TRANSACTIONS OF THE

AMERICAN MATHEMATICAL SOCIETY

Volume 352, Number 4 , Pages 1687-1708

S 0002-9947(99)02224-2

Article electronically published on November 17, 1999

\title{
PREHOMOGENEOUS VECTOR SPACES AND ERGODIC THEORY II
}

\author{
DAVE WITTE, AKIHIKO YUKIE, AND ROGER ZIERAU
}

\begin{abstract}
We apply M. Ratner's theorem on closures of unipotent orbits to the study of three families of prehomogeneous vector spaces. As a result, we prove analogues of the Oppenheim Conjecture for simultaneous approximation by values of certain alternating bilinear forms in an even number of variables and certain alternating trilinear forms in six and seven variables.
\end{abstract}

\section{INTRODUCTION}

Throughout this paper, $k$ is a field of characteristic zero. We first recall the definition of prehomogeneous vector spaces.

Definition (0.1). Let $G$ be a connected reductive group, $V$ a representation of $G$, and $\chi$ a non-trivial character of $G$, all defined over $k$. Then $(G, V, \chi)$ is called a prehomogeneous vector space if it satisfies the following properties.

(1) There exists a Zariski open orbit.

(2) There exists a non-zero polynomial $\Delta(x) \in k[V]$ such that $\Delta(g x)=\chi(g) \Delta(x)$.

Such $\Delta(x)$ is called a relative invariant polynomial. We define $V^{\text {ss }}=\{x \in V \mid$ $\Delta(x) \neq 0\}$ and call it the set of semi-stable points. If $(G, V, \chi)$ is an irreducible representation, the choice of $\chi$ is essentially unique and we may write $(G, V)$ as well. The theory of prehomogeneous vector spaces was initiated by Sato-Shintani [15] and Shintani [17]. If $(G, V)$ is irreducible, the classification is known (see [14]).

In this paper we consider the following three prehomogeneous vector spaces

(1) $G=\mathrm{GL}(6), V=\wedge^{3} k^{6}$,

(2) $G=\mathrm{GL}(1) \times \mathrm{GL}(7), V=\wedge^{3} k^{7}$,

(3) $G=\mathrm{GL}(2 n), V=\wedge^{2} k^{2 n}$.

We do not need the GL(1)-factor for cases (1) and (3), because having an extra GL(1)-factor does not change the orbit space $G_{k} \backslash V_{k}^{\text {ss }}$ for these cases.

For any algebraic group $G$, we denote the identity component in the Zariski topology by $G^{\circ}$. If $G$ is defined over a subfield of $\mathbb{R}$, we denote the identity component of $G_{\mathbb{R}}$ in the classical topology by $G_{\mathbb{R}+}^{\circ}$. Let $(G, V)$ be one of the prehomogeneous vector spaces (1)-(3). Let $H=\mathrm{SL}(n) \subset G(n=6,7)$ or $H=\mathrm{SL}(2 n)$. For $x \in V_{k}^{\mathrm{ss}}$, let $G_{x}$ be the stabilizer and $H_{x}=G_{x} \cap H$.

Received by the editors May 12, 1997.

1991 Mathematics Subject Classification. Primary 11J25; Secondary 22E30.

The first author was partially supported by NSF grant DMS-9214077; the second author was partially supported by NSF grant DMS-9401391; the third author was partially supported by NSF grant DMS-9303224. 
Consider the case $k=\mathbb{Q}$. Note that $H_{\mathbb{R}+}^{\circ}=H_{\mathbb{R}}$. Let $\Gamma \subset H_{\mathbb{R}+}^{\circ}=H_{\mathbb{R}}$ be an arithmetic lattice. The second author posed the following question in [20].

Question (0.5) [20]. (1) If $x \in V_{\mathbb{R}}^{\mathrm{ss}}$ is sufficiently irrational, is $H_{x \mathbb{R}+}^{\circ} \Gamma$ dense in $H_{\mathbb{R}}$ ?

(2) If (1) is true, find an explicit irrationality condition for (1) to be true.

We pointed out in [20] that for almost all $x \in V_{\mathbb{R}}^{\text {ss }}$ such that $H_{x \mathbb{R}}^{\circ}$ has a positive real rank, (1) is true by the Moore ergodicity theorem. The purpose of this paper is to consider the question (2) and interpret the result number theoretically. Our method is based on the following theorem due to Ratner.

Theorem (0.2) (Ratner). Let $G$ be a connected Lie group and $U$ a connected subgroup of $G$ generated by unipotent elements of $G$. Then given any lattice $\Gamma \subset G$ and $x \in G / \Gamma$, there exists a connected closed subgroup $U \subset F \subset G$ such that $\overline{U x \Gamma}=F x \Gamma$. Moreover, $F / F \cap x \Gamma x^{-1}$ has a finite invariant measure.

Note that in the above theorem, the definition of a lattice contains the condition that $G / \Gamma$ has a finite volume. The first statement was called Raghunathan's topological conjecture, and the second statement was proved by Ratner in conjunction with Raghunathan's topological conjecture. Raghunathan's topological conjecture was stated by Dani 2] for one dimensional unipotent groups and was generalized to groups generated by unipotent elements by Margulis [6], 7]. The proof for the general case was given by Ratner in a series of papers [9, [10], [11], [12]. For these, there is an excellent survey article by Ratner [13].

Note that in the above theorem, if $G$ is an algebraic group over $\mathbb{Q}$ and $\Gamma$ is an arithmetic lattice, the group $F$ becomes an algebraic group defined over $\mathbb{Q}$. For this, the reader should see Proposition (3.2) [16, pp. 321-322]. It is also proved in Proposition (3.2) [16, pp. 321-322] that the radical of $F$ is a unipotent subgroup. In [16], only one lattice is considered, but one can deduce the above statement for any lattice commensurable with the lattice in [16] by a simple argument using Ratner's theorem.

The following theorem is the main result of this paper. In statements (1)-(3) of the following theorem, we consider the prehomogeneous vector spaces (1)-(3) respectively. Let $W$ be the standard representation of GL(6), GL(7), or GL(2n). We identify $\wedge^{3} W$ and $\wedge^{2} W$ with $\wedge^{3}\left(W^{*}\right)^{*}$ and $\wedge^{2}\left(W^{*}\right)^{*}$ respectively. We define the notion of "sufficiently irrational" points in Definition (5.1)(1)-(3).

Theorem (6.15). Suppose $x \in V_{\mathbb{R}}^{\mathrm{ss}}$ is sufficiently irrational and the real rank of $H_{x \mathbb{R}}^{\circ}$ is positive.

(1) For any $y=\left(y_{i j k}\right) \in \wedge^{3} \mathbb{R}^{5}$ and $\epsilon>0$, there exists a $\mathbb{Z}$-basis $\left\{u_{1}, \cdots, u_{6}\right\}$ of $W_{\mathbb{Z}}^{*} \cong \mathbb{Z}^{6}$ such that

$$
\left|y_{i j k}-x\left(u_{i}, u_{j}, u_{k}\right)\right|<\epsilon
$$

for all $i<j<k \leq 5$.

(2) For any $y=\left(y_{i j k}\right) \in \wedge^{3} \mathbb{R}^{6}$ and $\epsilon>0$, there exists a $\mathbb{Z}$-basis $\left\{u_{1}, \cdots, u_{7}\right\}$ of $W_{\mathbb{Z}}^{*} \cong \mathbb{Z}^{7}$ such that

$$
\left|y_{i j k}-x\left(u_{i}, u_{j}, u_{k}\right)\right|<\epsilon
$$

for all $i<j<k \leq 6$. 
(3) For any $y=\left(y_{i j}\right) \in \wedge^{2} \mathbb{R}^{2 n-1}$ and $\epsilon>0$, there exists a $\mathbb{Z}$-basis $\left\{u_{1}, \cdots, u_{2 n}\right\}$ of $W_{\mathbb{Z}}^{*} \cong \mathbb{Z}^{2 n}$ such that

$$
\left|y_{i j}-x\left(u_{i}, u_{j}\right)\right|<\epsilon
$$

for all $i<j \leq 2 n-1$.

\section{The orbit SPACE $G_{k} \backslash V_{k}^{\mathrm{ss}}(1)$}

We describe the orbit space $G_{k} \backslash V_{k}^{\text {ss }}$ in $\S \S 1,2$. Let $W$ be as in the introduction. Throughout this paper, for a basis $\left\{e_{1}, e_{2}, \cdots\right\}$ of $W$, we use the notation $e_{i_{1} \cdots i_{k}}=$ $e_{i_{1}} \wedge \cdots \wedge e_{i_{k}}$.

For case (3), $G_{k} \backslash V_{k}^{\text {ss }}$ consists of a single point due to the well known fact that over any field two symplectic forms are equivalent. Let $e_{1}, \cdots, e_{2 n}$ be the standard coordinate vectors of $k^{2 n}$. Let $w=e_{1 n+1}+\cdots+e_{n 2 n}$. Then $G_{k} \backslash V_{k}^{\text {ss }}=G_{k} w$ and $G_{w}=G_{w}^{\circ}=H_{w}=H_{w}^{\circ}=\operatorname{Sp}(2 n)$.

We consider case (1) for the rest of this section. Let $G=\mathrm{GL}(6), W=k^{6}$, and $V=\wedge^{3} W$. It is known (see [14, p. 80]) that this is a prehomogeneous vector space. Let $\left\{e_{1}, \cdots, e_{6}\right\}$ be a basis of $W$. It is known (see [14]) that the orbit of

$$
w=e_{123}+e_{456}
$$

is Zariski open in $V$.

Let

$$
d(A, B)=\left(\begin{array}{cc}
A & 0 \\
0 & B
\end{array}\right)
$$

for $A, B \in \mathrm{M}(3,3)$. Then

$$
G_{w}^{\circ}=\{d(A, B) \mid A, B \in \mathrm{GL}(3), \operatorname{det} A=\operatorname{det} B=1\} \cong \mathrm{SL}(3) \times \mathrm{SL}(3)
$$

and $G_{w}$ is generated by $G_{w}^{\circ}$ and the element

$$
\tau=\left(\begin{array}{cc}
0 & I_{3} \\
I_{3} & 0
\end{array}\right)
$$

This implies that the following sequence

$$
1 \rightarrow G_{w}^{\circ} \rightarrow G_{w} \rightarrow \mathbb{Z} / 2 \mathbb{Z} \rightarrow 1
$$

which sends $\tau$ to the non-trivial element of $\mathbb{Z} / 2 \mathbb{Z}$ is exact. Moreover, this exact sequence is split. For these facts, the reader should see [14]. Note that the argument in 14] works over any ground field of characteristic zero.

For any algebraic group $G$ over $k$, let $\mathrm{H}^{1}(k, G)$ be the first Galois cohomology set. We choose the definition so that trivial classes are those of the form $\left\{g^{-1} g^{\sigma}\right\}_{\sigma \in \operatorname{Gal}(\bar{k} / k)}\left(g \in G_{\bar{k}}\right)$ and the cocycle condition is $h_{\sigma \tau}=h_{\tau} h_{\sigma}^{\tau}$ for a continuous map $\left\{h_{\sigma}\right\}_{\sigma \in \operatorname{Gal}(\bar{k} / k)}$ from $\operatorname{Gal}(\bar{k} / k)$ to $G_{\bar{k}}$.

For $x=g w \in V_{k}^{\text {ss }}$ where $g \in G_{\bar{k}}$, we define $c_{x}=\left\{g^{-1} g^{\sigma}\right\}_{\sigma \in \operatorname{Gal}(\bar{k} / k)} \in \mathrm{H}^{1}\left(k, G_{w}\right)$. This definition does not depend on the choice of $g$. Since $\mathrm{H}^{1}(k, G)=\{1\}$, by [4, p. 269],

$$
G_{k} \backslash V_{k}^{\mathrm{ss}} \ni x \rightarrow c_{x} \in \mathrm{H}^{1}\left(k, G_{w}\right)
$$

is bijective. 
Let $\mathfrak{E x}_{2}$ be the set of isomorphism classes of extensions $k^{\prime} / k$ of degree either one or two. By the split exact sequence (1.5), we get a surjective map

$$
\alpha_{V}: G_{k} \backslash V_{k}^{\mathrm{ss}} \rightarrow \mathrm{H}^{1}(k, \mathbb{Z} / 2 \mathbb{Z}) \cong \mathfrak{E x}_{2} .
$$

For $x \in G_{k} \backslash V_{k}^{\text {ss }}$, we denote the field corresponding to $\alpha_{V}(x)$ by $k(x)$. Let $k(\alpha)$ be the field generated by an element of the form $\alpha=\sqrt{\beta}$. We define $w_{\alpha}=g_{\alpha} w$ where

$$
g_{\alpha}=\left(\begin{array}{cccccc}
1 & 0 & 0 & 1 & 0 & 0 \\
\alpha & 0 & 0 & -\alpha & 0 & 0 \\
0 & 1 & 0 & 0 & 1 & 0 \\
0 & \alpha & 0 & 0 & -\alpha & 0 \\
0 & 0 & 1 & 0 & 0 & 1 \\
0 & 0 & \alpha & 0 & 0 & -\alpha
\end{array}\right) .
$$

Suppose $\alpha \notin k^{\times}$and $\sigma$ is the non-trivial element of $\operatorname{Gal}(k(\alpha) / k)$. It is easy to see that $g_{\alpha}^{\sigma}=g_{\alpha} \tau$. This implies that $\left(g_{\alpha} w\right)^{\sigma}=g_{\alpha} \tau w=g_{\alpha} w$. So $w_{\alpha} \in V_{k}^{\text {ss }}$ and $k\left(w_{\alpha}\right)=k(\alpha)$. Easy computations show that

$$
w_{\alpha}=e_{123}+\alpha^{2}\left(e_{156}-e_{246}+e_{345}\right) .
$$

We determine $G_{w_{\alpha}}^{\circ}$ rationally.

Proposition (1.10). If $\alpha \notin k^{\times}$, as an algebraic group over $k$,

$$
\begin{aligned}
G_{w_{\alpha}}^{\circ} & =\left\{g_{\alpha} d\left(A, A^{\sigma}\right) g_{\alpha}^{-1} \mid A \in \mathrm{SL}(3)_{k(\alpha)}\right\} \\
& \cong \mathrm{SL}(3)_{k(\alpha)} .
\end{aligned}
$$

Proof. In order to prove an isomorphism between two algebraic groups $G_{1}, G_{2}$ over $k$, it is enough to prove natural isomorphisms between the sets $G_{1 R}, G_{2 R}$ of $R$ rational points of $G_{1}, G_{2}$ for all $k$-algebras $R$. For this, the reader should see Theorem [8 p. 17].

Let $R$ be any $k$-algebra. For any finite Galois extension $k^{\prime} / k, \nu \in \operatorname{Gal}\left(k^{\prime} / k\right)$ acts on $k^{\prime} \otimes R$ by $(x \otimes r)^{\nu}=x^{\nu} \otimes r$. We define $R(\alpha)=k(\alpha) \otimes R$. Then

$$
G_{w_{\alpha} R}^{\circ}=\left\{g \in G_{w_{\alpha} R(\alpha)}^{\circ} \mid g^{\sigma}=g\right\} .
$$

Over $R(\alpha)$, we can express elements of $G_{w_{\alpha} R}^{\circ}$ as

$$
g_{\alpha} d(A, B) g_{\alpha}^{-1},
$$

where $A, B \in \mathrm{SL}(3)_{R(\alpha)}$. The element (1.11) is in $G_{w_{\alpha} R}^{\circ}$ if and only if

$$
\begin{aligned}
g_{\alpha} d(A, B) g_{\alpha}^{-1} & =g_{\alpha}^{\sigma} d\left(A^{\sigma}, B^{\sigma}\right)\left(g_{\alpha}^{\sigma}\right)^{-1} \\
& =g_{\alpha} \tau d\left(A^{\sigma}, B^{\sigma}\right) \tau g_{\alpha}^{-1} \\
& =g_{\alpha} d\left(B^{\sigma}, A^{\sigma}\right) g_{\alpha}^{-1} .
\end{aligned}
$$

This condition is satisfied if and only if $B=A^{\sigma}$. This proves the proposition.

Note that

$$
g_{\alpha} \tau g_{\alpha}^{-1}=\left(\begin{array}{cc}
I_{3} & \\
& -I_{3}
\end{array}\right)
$$

So the sequence

$$
1 \rightarrow G_{w_{\alpha}}^{\circ} \rightarrow G_{w_{\alpha}} \rightarrow \mathbb{Z} / 2 \mathbb{Z} \rightarrow 1
$$

is also a split exact sequence. 
By Lemma (1.4) [5, $\mathrm{H}^{1}\left(k, G_{w_{\alpha}}^{\circ}\right)$ is trivial for all $\alpha$. So by Lemma (1.12) [5], we get the following proposition.

Proposition (1.12). The map $\alpha_{V}$ induces a bijection $G_{k} \backslash V_{k}^{\mathrm{ss}} \cong \mathfrak{E x}_{2}$.

This gives us an interpretation of the expected density theorem from the zeta function theory of this case and the zeta function is a counting function of

$$
\zeta_{k(\alpha)}(2) \zeta_{k(\alpha)}(3)
$$

for quadratic extensions $k(\alpha)\left(\zeta_{k(\alpha)}(s)\right.$ is the Dedekind zeta function). However, we will not consider the zeta function in this paper. For the zeta function theory of prehomogeneous vector spaces, see [18], [19].

If $k=\mathbb{R}, G_{\mathbb{R}} \backslash V_{\mathbb{R}}^{\text {ss }}$ is represented by $w_{0}=w$ and

$$
w_{1}=e_{123}-e_{156}+e_{246}-e_{345} .
$$

As in [14, we define a map $D_{3}: \wedge^{3} W \rightarrow \wedge^{2} W \otimes W$ by

$$
D_{3}\left(v_{1} \wedge v_{2} \wedge v_{3}\right)=v_{2} \wedge v_{3} \otimes v_{1}-v_{1} \wedge v_{3} \otimes v_{2}+v_{1} \wedge v_{2} \otimes v_{3}
$$

for $v_{1}, v_{2}, v_{3} \in W$.

For $x \in V_{k}$, we define

$$
S_{x}=x \wedge D_{3}(x) \in \wedge^{5} W \otimes W \cong W^{*} \otimes W \cong \operatorname{Hom}(W, W),
$$

where $x \wedge(y \otimes z)=(x \wedge y) \otimes z$.

It is proved in [14 p. 80] that there is a relative invariant polynomial $\Delta(x)$ of degree four such that $S_{x}^{2}=\Delta(x) I_{W}$ where $I_{W}$ is the identity map of $W$. This implies that

$$
\Delta(g x)=(\operatorname{det} g)^{2} \Delta(x) .
$$

Since $S_{w}$ has eigenvalues \pm 1 (see [14 p. 80]), $\Delta(w)=1$. Also since ch $k=0$ and $\Delta(w) \in k$, we may assume that $\Delta \in k[V]$. Note that $\operatorname{det} g_{\alpha}=-8 \alpha$. So $\Delta\left(w_{\alpha}\right)=64 \alpha^{2} \Delta(w)=64 \alpha^{2}$. Therefore, this gives us the following characterization of the field $k(x)$.

Proposition (1.17). For $x \in V_{k}^{\text {ss }}$, the field $k(x)$ is generated by eigenvalues of $S_{x}$.

Let $E_{x 1}, E_{x 2}$ be the eigenspaces of $S_{x}$ for the eigenvalues $\pm \Delta(x)^{\frac{1}{2}}$. It is known [14] that $\operatorname{dim} E_{x 1}=\operatorname{dim} E_{x 2}=3$. Let $\operatorname{Gr}(3,6)$ be the Grassmann of 3-dimensional subspaces of $W$. Let

$$
X=(\mathbb{Z} / 2 \mathbb{Z}) \backslash(\operatorname{Gr}(3,6) \times \operatorname{Gr}(3,6)),
$$

where $\mathbb{Z} / 2 \mathbb{Z}$ acts by permuting two factors.

Definition (1.19). $\operatorname{Gr}(x)=\left(\left[E_{x 1}\right],\left[E_{x 2}\right]\right) \in X$.

Since we are assuming ch $k=0, k$-rational points of $X$ are points which are set theoretically fixed by any $\sigma \in \operatorname{Gal}(\bar{k} / k)$. If $x \in V_{k}^{\text {ss }}, E_{x 1}, E_{x 2}$ are conjugate. So $\operatorname{Gr}(x)$ is a $k$-rational point of $X$. The following proposition is obvious.

Proposition (1.20). If $x \in V_{\bar{k}}^{\mathrm{ss}}$ and $\sigma \in \operatorname{Gal}(\bar{k} / k), \operatorname{Gr}\left(x^{\sigma}\right)=\operatorname{Gr}(x)^{\sigma}$.

For general $x \in V^{\mathrm{ss}}$, we cannot distinguish $E_{x 1}$ and $E_{x 2}$. But for later purposes, we choose $E_{w 1}$ and $E_{w 2}$ so that $E_{w 1}$ is spanned by $e_{1}, e_{2}, e_{3}$ and $E_{w 2}$ is spanned by $e_{4}, e_{5}, e_{6}$. It is proved in [14, p. 80] that $E_{w 1}\left(\right.$ resp. $\left.E_{w 2}\right)$ is the eigenspace of $S_{w}$ for the eigenvalue 1 (resp. -1 ). 


\section{The ORBIT SPACE $G_{k} \backslash V_{k}^{\mathrm{ss}}(2)$}

We describe the orbit space $G_{k} \backslash V_{k}^{\text {ss }}$ for case (2) in this section. Since this case has something to do with octonion algebras, we briefly recall the CayleyDickson process. Octonian algebras are often referred to as Cayley algebras also. For a reference, see [3, pp. 101-110] for example. Note that even though [3] assumes $k=\mathbb{R}$, the argument for the Cayley-Dickson process works over any field of characteristic zero.

Definition (2.1). A normed $k$-algebra is a not necessarily associative finite dimensional $k$-algebra $A$ with multiplicative unit 1 , equipped with a non-degenerate symmetric bilinear form $\langle x, y\rangle$ for $x, y \in A$ such that the associated square norm $\|x\|=\langle x, x\rangle$ satisfies the multiplicative property

$$
\|x y\|=\|x\|\|y\| \text {. }
$$

If $A$ is a normed $k$-algebra, we denote the span of 1 by $\operatorname{Re}(A)$ and its orthogonal complement $\{x \in A \mid\langle 1, x\rangle=0\}$ by $\operatorname{Im}(A)$. Any $x \in A$ has a unique decomposition $x=x_{1}+x_{2}$ with $x_{1} \in \operatorname{Re}(A), x_{2} \in \operatorname{Im}(A)$. We denote $\operatorname{Re}(x)=x_{1}, \operatorname{Im}(x)=x_{2}$. We define the conjugation by $\bar{x}=x_{1}-x_{2}$. So $\operatorname{Re}(x)=\frac{1}{2}(x+\bar{x})$.

Given a normed $k$-algebra $A$, we make two new normed $k$-algebras $A( \pm)$ as follows. As a vector space,

$$
A( \pm)=A \oplus A
$$

We define the multiplication and the norm by

$$
\begin{aligned}
& (a, b)(c, d)=(a c \mp \bar{d} b, d a+b \bar{c}), \\
& \|(a, b)\|=\|a\| \pm\|b\| .
\end{aligned}
$$

Then we define

$$
\langle(a, b),(c, d)\rangle=\frac{1}{2}(\|(a, b)+(c, d)\|-\|(a, b)\|-\|(c, d)\|) .
$$

The algebra $A( \pm)$ becomes a normed $k$-algebra by the above product and the bilinear form. We use the notation $a+b \epsilon$ for $(a, b)$. Note that if $k$ contains $\sqrt{-1}$, $\epsilon \rightarrow \sqrt{-1} \epsilon$ induces an isomorphism $A(+) \rightarrow A(-)$.

For a normed $k$-algebra $A$, we define $[x, y, z]=(x y) z-x(y z)$ for $x, y, z \in A$. This is called the associator. If the associator is alternating, $A$ is called an alternative algebra. It is known that if $A$ is commutative, $A( \pm)$ is associative, and if $A$ is associative, $A( \pm)$ is alternative. It is known that the norm of $A( \pm)$ is compatible with the product if and only if $A$ is associative. The above process is called the Cayley-Dickson process. It is easy to see that

$$
\operatorname{Im}(a+b \epsilon)=\operatorname{Im}(a)+b \epsilon, \quad \overline{a+b \epsilon}=\bar{a}-b \epsilon .
$$

The following lemma is proved in [3].

Lemma (2.4). (1) $\overline{x y}=\bar{y} \bar{x}$,

(2) $\langle x, y\rangle=\operatorname{Re}(x \bar{y})$.

(3) $\|x\|=x \bar{x}$.

If $A, B$ are normed $k$-algebras, a homomorphism $\phi: A \rightarrow B$ is a $k$-linear map such that $\phi(1)=1, \phi(x y)=\phi(x) \phi(y)$, and $\|\phi(x)\|=\|x\|$. The third condition 
implies $\langle\phi(x), \phi(y)\rangle=\langle x, y\rangle$. So $\phi(\operatorname{Im}(A)) \subset \operatorname{Im}(B)$. Suppose $x, y \in \operatorname{Im}(A)$. Then $\langle x, y\rangle=\operatorname{Re}(x \bar{y})=-\operatorname{Re}(x y)$. So

$$
\begin{aligned}
-\operatorname{Re}(\phi(x) \phi(y)) & =\langle\phi(x), \phi(y)\rangle=\langle x, y\rangle=-\operatorname{Re}(x y), \\
\phi(\operatorname{Im}(x y)) & =\phi(x y-\operatorname{Re}(x y))=\phi(x) \phi(y)-\operatorname{Re}(x y) \\
& =\phi(x) \phi(y)-\operatorname{Re}(\phi(x) \phi(y))=\operatorname{Im}(\phi(x) \phi(y)) .
\end{aligned}
$$

Conversely, $\phi$ is a homomorphism if the above conditions are satisfied. So we have proved the following proposition.

Proposition (2.5). A k-linear map $\phi: A \rightarrow B$ is a homomorphism if and only if

$$
\phi(1)=1, \phi(\operatorname{Im}(x y))=\operatorname{Im}(\phi(x) \phi(y)),\langle\phi(x), \phi(y)\rangle=\langle x, y\rangle
$$

for all $x, y \in \operatorname{Im}(A)$.

It is easy to see that

$$
\begin{aligned}
& k(+) \cong\left\{\left(\begin{array}{cc}
a & -b \\
b & a
\end{array}\right) \mid a, b \in k\right\}, \\
& k(+)(-) \cong \mathrm{M}(2,2)_{k} .
\end{aligned}
$$

For $k(+), \epsilon=\left(\begin{array}{cc}0 & -1 \\ 1 & 0\end{array}\right)$, and the conjugation is

$$
\left(\begin{array}{cc}
a & -b \\
b & a
\end{array}\right) \rightarrow\left(\begin{array}{cc}
a & b \\
-b & a
\end{array}\right)
$$

For $k(+)(-), \epsilon=\left(\begin{array}{cc}1 & 0 \\ 0 & -1\end{array}\right)$, and the conjugation is

$$
\left(\begin{array}{ll}
a & b \\
c & d
\end{array}\right) \rightarrow\left(\begin{array}{cc}
d & -b \\
-c & a
\end{array}\right)
$$

Therefore, $\operatorname{Re}(x)=\frac{1}{2} \operatorname{tr}(x)$ in both cases and the norm is the determinant.

We define $\mathbb{H}=k(+)(+), \mathbb{O}=\mathbb{H}(+)$, and $\widetilde{\mathbb{O}}=\mathrm{M}(2,2)(+)$. $\mathbb{O}$ is called the non-split octonion algebra (if $k$ does not contain $\sqrt{-1}$ ), and $\widetilde{\mathbb{O}}$ is called the split octonion algebra.

We consider properties of the split octonion algebra $\widetilde{\mathbb{O}}$. Let

$$
C(x, y, z)=\langle x, y z\rangle
$$

for $x, y, z \in \widetilde{\mathbb{O}}$. Suppose $x=x_{1}+x_{2} \epsilon, y=y_{1}+y_{2} \epsilon$, and $z=z_{1}+z_{2} \epsilon$.

\section{Lemma (2.8).}

$$
C(x, y, z)=\frac{1}{2} \operatorname{tr}\left(x_{1}\left(\bar{z}_{1} \bar{y}_{1}-\bar{y}_{2} z_{2}\right)+\left(\bar{y}_{1} \bar{z}_{2}+z_{1} \bar{y}_{2}\right) x_{2}\right) .
$$

Proof. Since $y z=y_{1} z_{1}-\bar{z}_{2} y_{2}+\left(z_{2} y_{1}+y_{2} \bar{z}_{1}\right) \epsilon$,

$$
\begin{aligned}
\overline{y z}= & \bar{z}_{1} \bar{y}_{1}-\bar{y}_{2} z_{2}-\left(z_{2} y_{1}+y_{2} \bar{z}_{1}\right) \epsilon, \\
x(\overline{y z})= & x_{1}\left(\bar{z}_{1} \bar{y}_{1}-\bar{y}_{2} z_{2}\right)+\left(\bar{y}_{1} \bar{z}_{2}+z_{1} \bar{y}_{2}\right) x_{2} \\
& +\left(-\left(z_{2} y_{1}+y_{2} \bar{z}_{1}\right) x_{1}+x_{2}\left(y_{1} z_{1}-\bar{z}_{2} y_{2}\right)\right) \epsilon .
\end{aligned}
$$


Therefore,

$$
\begin{aligned}
C(x, y, z) & =\operatorname{Re}(x(\overline{y z})) \\
& =\operatorname{Re}\left(x_{1}\left(\bar{z}_{1} \bar{y}_{1}-\bar{y}_{2} z_{2}\right)+\left(\bar{y}_{1} \bar{z}_{2}+z_{1} \bar{y}_{2}\right) x_{2}\right) \\
& =\frac{1}{2} \operatorname{tr}\left(x_{1}\left(\bar{z}_{1} \bar{y}_{1}-\bar{y}_{2} z_{2}\right)+\left(\bar{y}_{1} \bar{z}_{2}+z_{1} \bar{y}_{2}\right) x_{2}\right) .
\end{aligned}
$$

Note that $\operatorname{tr}(x)=\operatorname{tr}(\bar{x})$ for $x \in \mathrm{M}(2,2)$. Also if $x, y, z \in \operatorname{Im}(\widetilde{\mathbb{O}}), x_{1}+\bar{x}_{1}=$ $y_{1}+\bar{y}_{1}=z_{1}+\bar{z}_{1}=0$. Therefore, the above lemma and a straightforward argument shows that $C(x, y, z)$ is an element of $\wedge^{3} \operatorname{Im}(\widetilde{\mathbb{O}})^{*}$ (see (6.60) [3, p. 113] also).

Let $E_{i j}$ be the $2 \times 2$ matrix whose $(i, j)$-entry is 1 and other entries are zero. Let

$$
\begin{aligned}
& f_{1}=\left(\begin{array}{cc}
1 & 0 \\
0 & -1
\end{array}\right), f_{2}=E_{12}, f_{3}=E_{11} \epsilon, f_{4}=-E_{21} \epsilon, \\
& f_{5}=-E_{21}, f_{6}=E_{22} \epsilon, f_{7}=E_{12} \epsilon .
\end{aligned}
$$

Then $\left\{f_{1}, \cdots, f_{7}\right\}$ is a basis for $\operatorname{Im}(\widetilde{\mathbb{O}})$. Let $e_{1}, \cdots, e_{7}$ be the dual basis. Straightforward computations (35 computations for (2)) using the above lemma shows the following proposition and the proof is left to the reader.

Proposition (2.10). (1) Suppose

$$
x=\sum_{i=1}^{7} x_{i} f_{i}=\left(\begin{array}{cc}
x_{1} & x_{2} \\
-x_{5} & -x_{1}
\end{array}\right)+\left(\begin{array}{cc}
x_{3} & x_{7} \\
-x_{4} & x_{6}
\end{array}\right) \epsilon .
$$

Then $\|x\|=-x_{1}^{2}+x_{2} x_{5}+x_{3} x_{6}+x_{4} x_{7}$.

(2) $C=\frac{1}{2}\left(e_{234}+e_{567}+e_{125}+e_{136}+e_{147}\right)$.

Let $W=\operatorname{Im}(\widetilde{\mathbb{O}})^{*}$. We choose the above basis $\left\{e_{1}, \cdots, e_{7}\right\}$. Let $G=\operatorname{GL}(1) \times$ $\operatorname{GL}(W), V=\wedge^{3} W$. The GL(1)-factor acts by the usual multiplication. We need this factor for number theoretic reasons unlike case (1). It is known 14 p. 83-87] that this is a prehomogeneous vector space and the orbit of

$$
w=e_{234}+e_{567}+e_{125}+e_{136}+e_{147}
$$

is Zariski open.

We use the operation $D_{3}$ defined in (1.14) again. For $x \in V$, we define

$$
S_{x}=x(\wedge, \otimes) D_{3}(x)(\wedge, \otimes) D_{3}(x) \in \wedge^{7} W \otimes W \otimes W \cong W \otimes W,
$$

where $(\wedge, \otimes)$ means the wedge product for the first factor and the tensor product for the second factor. Let $\phi: W \otimes W \rightarrow \operatorname{Sym}^{2} W$ be the canonical map.

Definition (2.13). $Q_{x}=\phi\left(S_{x}\right)$.

We regard $Q_{x}$ and $x$ as elements of $\operatorname{Sym}^{2}\left(W^{*}\right)^{*} \cong \operatorname{Sym}^{2} \operatorname{Im}(\widetilde{\mathbb{O}})^{*}$ and $\wedge^{3}\left(W^{*}\right)^{*}$ respectively. So $Q_{x}$ is a quadratic polynomial on $W^{*}$ and $x$ is an alternating trilinear form on $W^{*}$. We use the same notation $Q_{x}$ for the associated bilinear form on $W^{*}$. We define the action of $\operatorname{GL}(W)$ on $W^{*}$ by $g f(v)=f\left(g^{-1} v\right)$. In this way, we identify $\operatorname{GL}(W)$ and $\operatorname{GL}\left(W^{*}\right)$. Note that if we use bases for $W, W^{*}$ which are dual to each other and identify $\mathrm{GL}(W), \mathrm{GL}\left(W^{*}\right)$ with the set of $7 \times 7$ matrices, $g \in \mathrm{GL}(7)$ corresponds to ${ }^{t} g^{-1}$ by this identification. It is easy to see that

$$
Q_{(t, g) x}=t^{3}(\operatorname{det} g) g Q_{x}
$$


(we are taking the determinant considering $g \in \mathrm{GL}(W)$ ).

One can compute $Q_{x}$ for $x=w$ and

$$
w^{\prime}=e_{234}+e_{346}+e_{127}-e_{145}
$$

by rather long and tedious computations (it takes about 10 hours to do so manually), and the result is

$$
\begin{aligned}
& Q_{w}=6\left(-e_{1}^{2}+e_{2} e_{5}+e_{3} e_{6}+e_{4} e_{7}\right), \\
& Q_{w^{\prime}}=6\left(-e_{1} e_{4}+e_{2} e_{3}\right) .
\end{aligned}
$$

We verified the above computation by the software "MAPLE" [1 also. For example, to compute $Q_{w^{\prime}}$, we associate a differential form $d x_{2} \wedge d x_{3} \wedge d x_{4}+\cdots$ to $w^{\prime}$ and the input is as follows.

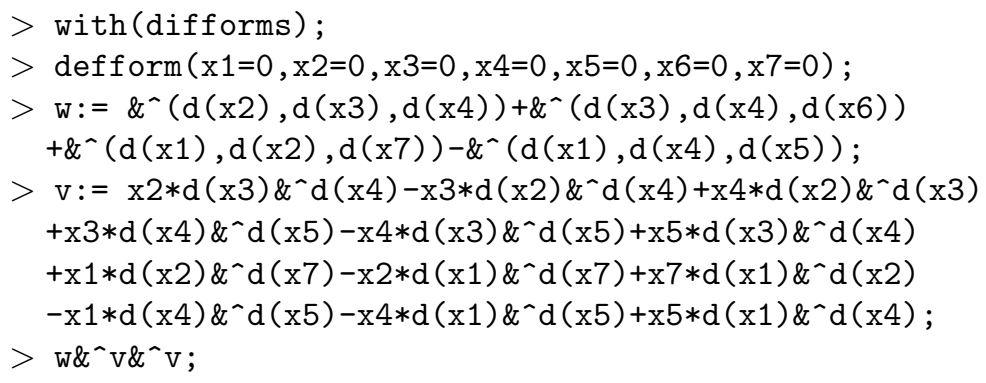

Since $Q_{w}$ is non-degenerate, the discriminant of $Q_{x}$ is a non-zero relative invariant polynomial of degree 21 and this reproves the existence of a relative invariant polynomial. Since $Q_{w}$ is irreducible, $Q_{x}$ is irreducible as a polynomial of $v \in W^{*}$. If $Q_{x}$ is divisible by a non-constant polynomial $p(x)$ of $x, p(x)$ is a relative invariant polynomial. Since $Q_{w^{\prime}}$ is degenerate, $p\left(w^{\prime}\right)=0$. But since $Q_{w^{\prime}}$ is non-zero, this is a contradiction. So we get the following proposition.

Proposition (2.17). As a polynomial of $(x, v) \in V \oplus W^{*}, Q_{x}(v)$ is irreducible.

If $A$ is a normed $k$-algebra, $\operatorname{Re}(A)$ is contained in the center of $A$. So the structure of $A$ is determined by its restriction to $\operatorname{Im}(A)$. On $W^{*}$, we define a product structure $(\cdot)_{x}$ depending on $x$ by the equation

$$
3 x\left(v_{1}, v_{2}, v_{3}\right)=Q_{x}\left(v_{1},\left(v_{2} \cdot v_{3}\right)_{x}\right)
$$

for all $v_{1}, v_{2}, v_{3} \in W^{*}$. It is known [14, p. 86] that $(G, V)$ has a relative invariant polynomial $\Delta(x)$ of degree seven. Since ch $k=0$, we may assume that $\Delta(x) \in k[V]$ and $\Delta(w)=6$. Clearly, $\Delta((t, g) x)=t^{7}(\operatorname{det} g)^{3} \Delta(x)$. For $x \in V_{k}^{\text {ss }}$ and $v \in W_{k}^{*}$, we define

$$
\|v\|_{x}=\Delta(x)^{-1} Q_{x}(v) .
$$

Definition (2.20). $\mathbb{O}_{x}$ is the algebra $k \oplus W^{*}$ such that $W^{*}=\operatorname{Im}\left(\mathbb{O}_{x}\right)$. The norm is defined by $\|v\|=\|v\|_{x}$ for $v \in W^{*}$, and the product $v_{1} v_{2}$ for $v_{1}, v_{2} \in W^{*}$ is defined by

$$
\operatorname{Re}\left(v_{1} v_{2}\right)=-\Delta(x)^{-1} Q_{x}\left(v_{1}, v_{2}\right), \operatorname{Im}\left(v_{1} v_{2}\right)=\left(v_{1} \cdot v_{2}\right)_{x} .
$$

If $x=w$, the product structure and the norm of $\mathbb{O}_{w}$ coincide with those of $\widetilde{\mathbb{O}}$ by Proposition (2.10). Therefore, $\mathbb{O}_{w} \cong \widetilde{\mathbb{O}}$ and is a normed $k$-algebra. Suppose 
$x, y \in V_{k}^{\mathrm{ss}},(t, g) \in G_{\bar{k}}$, and $y=(t, g) x$. Then we define $m_{x, y,(t, g)}: W^{*} \rightarrow W^{*}$ by

$$
m_{x, y,(t, g)}(v)=t^{2}(\operatorname{det} g) g v
$$

for $v \in W^{*}$. Note that we are taking the determinant of $g$ considering $g \in \mathrm{GL}(W)$.

Proposition (2.22). (1) For all $x \in V_{k}^{\mathrm{ss}}, \mathbb{O}_{x}$ is a normed $k$-algebra and is a $k$-form of $\widetilde{\mathbb{O}}$.

(2) If $x, y \in V_{k}^{\mathrm{ss}},(t, g) \in G_{\bar{k}}$, and $y=(t, g) x, m_{x, y,(t, g)}$ is an isomorphism of normed $\bar{k}$-algebras from $\mathbb{O}_{x \bar{k}}$ to $\mathbb{O}_{y \bar{k}}$.

Proof. Let $m=m_{x, y,(t, g)}$. Then

$$
\begin{aligned}
Q_{y}\left(g v_{1},\left(m\left(v_{2}\right) \cdot m\left(v_{3}\right)\right)_{y}\right) & =t^{4}(\operatorname{det} g)^{2} Q_{y}\left(g v_{1},\left(g v_{2} \cdot g v_{3}\right)_{y}\right) \\
& =3 t^{4}(\operatorname{det} g)^{2} y\left(g v_{1}, g v_{2}, g v_{3}\right) \\
& =3 t^{4}(\operatorname{det} g)^{2} g^{-1} y\left(v_{1}, v_{2}, v_{3}\right) \\
& =3 t^{5}(\operatorname{det} g)^{2} x\left(v_{1}, v_{2}, v_{3}\right) \\
& =t^{5}(\operatorname{det} g)^{2} Q_{x}\left(v_{1},\left(v_{2} \cdot v_{3}\right)_{x}\right) \\
& =t^{2} \operatorname{det} g Q_{y}\left(g v_{1}, g\left(v_{2} \cdot v_{3}\right)_{x}\right) \\
& =Q_{y}\left(g v_{1}, m\left(\left(v_{2} \cdot v_{3}\right)_{x}\right)\right.
\end{aligned}
$$

for all $v_{1}, v_{2}, v_{3} \in W^{*}$.

Therefore, $\left(m\left(v_{2}\right) \cdot m\left(v_{3}\right)\right)_{y}=m\left(v_{2} \cdot v_{3}\right)_{x}$. Since

$$
\begin{aligned}
\Delta(y)^{-1} Q_{y}\left(m\left(v_{1}\right), m\left(v_{2}\right)\right) & =\left(t^{7}(\operatorname{det} g)^{3}\right)^{-1} t^{4}(\operatorname{det} g)^{2} \Delta(x)^{-1} Q_{y}\left(g v_{1}, g v_{2}\right) \\
& =\left(t^{3} \operatorname{det} g\right)^{-1} \Delta(x)^{-1} t^{3} \operatorname{det} g Q_{x}\left(v_{1}, v_{2}\right) \\
& =\Delta(x)^{-1} Q_{x}\left(v_{1}, v_{2}\right)
\end{aligned}
$$

for all $v_{1}, v_{2} \in W^{*}, m$ preserves the norm also.

Since $\mathbb{O}_{w}$ is a normed $k$-algebra, this proves both (1), (2).

Let $\mathfrak{O}$ be the set of $k$-isomorphism classes of $k$-forms of $\widetilde{\mathbb{O}}$. We regard $\operatorname{Aut}(\widetilde{\mathbb{O}}) \subset$ $\operatorname{GL}(\operatorname{Im}(\widetilde{\mathbb{O}}))$.

Lemma (2.23). If $g \in \operatorname{Aut}(\widetilde{\mathbb{O}}), \operatorname{det} g=1$.

Proof. Since $Q_{w}\left(g v_{1}, g v_{2}\right)=Q_{w}\left(v_{1}, v_{2}\right)$ for all $v_{1}, v_{2} \in W^{*},(\operatorname{det} g)^{2}=1$. Since $g\left(v_{1} \cdot v_{2}\right)_{w}=\left(g v_{1} \cdot g v_{2}\right)_{w}, g w=w$ by (2.18). It is proved in [14, p. 86] that if $g \in G_{w} \cap \mathrm{GL}(W),(\operatorname{det} g)^{3}=1$. This implies $\operatorname{det} g=(\operatorname{det} g)^{3} /(\operatorname{det} g)^{2}=1$.

Proposition (2.24). The map

$$
\alpha_{V}: G_{k} \backslash V_{k}^{\mathrm{ss}} \ni x \rightarrow \mathbb{O}_{x} \in \mathfrak{O}
$$

is well defined and is bijective.

Proof. If $x, y \in V_{k}^{\text {ss }},(t, g) \in G_{k}$, and $y=(t, g) x$, then $m_{x, y,(t, g)}$ is a $k$-isomorphism. Therefore, the above map is well defined. Suppose $x, y \in V_{k}^{\text {ss }}$ and $g^{\prime}: \mathbb{O}_{x} \rightarrow \mathbb{O}_{y}$ is a $k$-isomorphism. Regarding $g^{\prime} \in \mathrm{GL}(W)_{k}$ (by considering the contragredient representation), let $t=\left(\operatorname{det} g^{\prime}\right)^{3}, g=\left(\operatorname{det} g^{\prime}\right)^{-1} g^{\prime}$. Then $(t, g) \in G_{k}$ and $t^{2} \operatorname{det} g g=$ 
$g^{\prime}$. Since $\Delta(x)^{-1} Q_{x}\left(v_{1}, v_{2}\right)=\Delta(y)^{-1} Q_{y}\left(g^{\prime} v_{1}, g^{\prime} v_{2}\right)$ for all $v_{1}, v_{2} \in W^{*}$, by a similar argument as in Proposition $(2.22), Q_{(t, g)^{-1} y}=Q_{x}$. So

$$
\begin{aligned}
3 t^{4}(\operatorname{det} g)^{2} g^{-1} y\left(v_{1}, v_{2}, v_{3}\right) & =3 y\left(g v_{1}, g^{\prime} v_{2}, g^{\prime} v_{3}\right) \\
& =Q_{y}\left(g v_{1},\left(g^{\prime} v_{2} \cdot g^{\prime} v_{3}\right)_{y}\right) \\
& =Q_{y}\left(g v_{1}, g^{\prime}\left(v_{2} \cdot v_{3}\right)_{x}\right) \\
& =t^{2} \operatorname{det} g Q_{y}\left(g v_{1}, g\left(v_{2} \cdot v_{3}\right)_{x}\right) \\
& =t^{5}(\operatorname{det} g)^{2} Q_{(t, g)^{-1}}\left(v_{1},\left(v_{2} \cdot v_{3}\right)_{x}\right) \\
& =t^{5}(\operatorname{det} g)^{2} Q_{x}\left(v_{1},\left(v_{2} \cdot v_{3}\right)_{x}\right) \quad \text { by the above remark } \\
& =3 t^{5}(\operatorname{det} g)^{2} x\left(v_{1}, v_{2}, v_{3}\right)
\end{aligned}
$$

for all $v_{1}, v_{2}, v_{3} \in W^{*}$.

Therefore, $y=(t, g) x \in G_{k} x$.

Let $A$ be a $k$-form of $\widetilde{\mathbb{O}}$ corresponding to a cohomology class $c \in \mathrm{H}^{1}(k, \operatorname{Aut}(\widetilde{\mathbb{O}}))$. Suppose $c=\left\{h_{\sigma}\right\}_{\sigma}$. Then

$$
\operatorname{Im}(A)_{k}=\left\{v \in \operatorname{Im}(\widetilde{\mathbb{O}})_{\bar{k}} \mid h_{\sigma} v^{\sigma}=v \text { for all } \sigma \in \operatorname{Gal}(\bar{k} / k)\right\} .
$$

Since $\operatorname{Aut}(\widetilde{\mathbb{O}}) \subset \mathrm{GL}(\operatorname{Im}(\widetilde{\mathbb{O}}))$ and $\mathrm{H}^{1}(k, \operatorname{GL}(\operatorname{Im}(\widetilde{\mathbb{O}})))$ is trivial, there exists $g^{\prime} \in$ $\operatorname{GL}(\widetilde{\mathbb{O}})_{\bar{k}}$ such that $c=\left\{g^{\prime-1} g^{\prime \sigma}\right\}_{\sigma}$. Then

$$
\begin{aligned}
\operatorname{Im}(A)_{k} & =\left\{v \in \operatorname{Im}(\widetilde{\mathbb{O}})_{\bar{k}} \mid g^{\prime-1} g^{\prime \sigma} v^{\sigma}=v \text { for all } \sigma \in \operatorname{Gal}(\bar{k} / k)\right\} \\
& =\left\{v \in \operatorname{Im}(\widetilde{\mathbb{O}})_{\bar{k}} \mid g^{\prime \sigma} v^{\sigma}=g^{\prime} v \text { for all } \sigma \in \operatorname{Gal}(\bar{k} / k)\right\} \\
& =\left\{v \in \operatorname{Im}(\widetilde{\mathbb{O}})_{\bar{k}} \mid g^{\prime} v \in \operatorname{Im}(\widetilde{\mathbb{O}})_{k}\right\} .
\end{aligned}
$$

Let $t=\left(\operatorname{det} g^{\prime}\right)^{3}$ and $g=\left(\operatorname{det} g^{\prime}\right)^{-1} g^{\prime}$. Then $(t, g) \in G_{\bar{k}}$. By Lemma (2.23),

$$
(t, g)^{-1}(t, g)^{\sigma}=\left(1, g^{\prime-1} g^{\prime \sigma}\right) \in G_{w \bar{k}} .
$$

So this defines a cohomology class in $\mathrm{H}^{1}\left(k, G_{w}\right)$. This implies that $x=(t, g) w \in$ $V_{k}^{\text {ss }}$. Since $g^{\prime}=m_{w, x, t, g)}, g^{\prime}$ induces an isomorphism from $\operatorname{Im}(\widetilde{\mathbb{O}})_{\bar{k}}$ to $\operatorname{Im}\left(\mathbb{O}_{x}\right)_{\bar{k}}$. Therefore, $A \cong \mathbb{O}_{x}$.

Remark (2.25). It is proved in [14, 4 4 that $G_{k} \backslash V_{k}^{\text {ss }} \cong \mathrm{H}^{1}(k$, Aut $(\widetilde{\mathbb{O}}))$. So the credit for the existence of a bijective correspondence between $G_{k} \backslash V_{k}^{\text {ss }}$ and $\mathfrak{O}$ should go to Sato-Kimura [14] and Igusa [4. However, we constructed $\mathbb{O}_{x} \in \mathfrak{O}$ for $x \in V_{k}^{\text {ss }}$, and the fact that this particular correspondence is bijective still required a proof. The operator $D_{3}$ was considered in [14]. The fact that the stabilizer of $w$ is a group of type $G_{2}$ at least goes back to [14].

For the rest of this section, we describe the orbit space $G_{\mathbb{R}} \backslash V_{\mathbb{R}}^{\text {ss }} \cong \mathrm{GL}(W)_{\mathbb{R}} \backslash V_{\mathbb{R}}^{\text {ss }}$. We will show that $G_{\mathbb{R}} \backslash V_{\mathbb{R}}^{\text {ss }}$ consists of two orbits corresponding to $\mathbb{O}, \widetilde{\mathbb{O}}$.

It is known that $\mathbb{O}, \widetilde{\mathbb{O}}$ are the only $\mathbb{R}$-forms of $\widetilde{\mathbb{O}}$. We can identify $\mathbb{H}$ with $\mathbb{C}(+)$ by

$$
a+b i+c j+d k \rightarrow(a+b i)+(c+d i) \epsilon .
$$

So $\mathbb{H} \otimes \mathbb{C}$ and $\mathrm{M}(2,2)_{\mathbb{C}}$ are isomorphic by the map

$$
a+b i+c j+d k \rightarrow\left(\begin{array}{cc}
a+\sqrt{-1} c & -b+\sqrt{-1} d \\
b+\sqrt{-1} d & a-\sqrt{-1} c
\end{array}\right)
$$

for $a, b, c, d \in \mathbb{C}($ see $(2.6))$. 
Therefore, by considering $\mathbb{O}=\mathbb{H}(+), \widetilde{\mathbb{O}}=\mathrm{M}(2,2)(+), \mathbb{O} \otimes \mathbb{C}$ and $\widetilde{\mathbb{O}} \otimes \mathbb{C}$ are isomorphic by the map

$$
\begin{aligned}
& \phi\left(\left(y_{1} i+y_{2} j+y_{3} k\right)+\left(y_{4}+y_{5} i+y_{6} j+y_{7} k\right) \epsilon\right) \\
& =\left(\begin{array}{cc}
\sqrt{-1} y_{2} & -y_{1}+\sqrt{-1} y_{3} \\
y_{1}+\sqrt{-1} y_{3} & -\sqrt{-1} y_{2}
\end{array}\right)+\left(\begin{array}{cc}
y_{4}+\sqrt{-1} y_{6} & -y_{5}+\sqrt{-1} y_{7} \\
y_{5}+\sqrt{-1} y_{7} & y_{4}-\sqrt{-1} y_{6}
\end{array}\right) \epsilon .
\end{aligned}
$$

Let $y=\left(y_{1} i+y_{2} j+y_{3} k\right)+\left(y_{4}+y_{5} i+y_{6} j+y_{7} k\right) \epsilon$. We define

$$
g_{1}=\left(\begin{array}{ccccccc}
0 & -1 & 0 & 0 & -1 & 0 & 0 \\
\sqrt{-1} & 0 & 0 & 0 & 0 & 0 & 0 \\
0 & \sqrt{-1} & 0 & 0 & -\sqrt{-1} & 0 & 0 \\
0 & 0 & 1 & 0 & 0 & 1 & 0 \\
0 & 0 & 0 & -1 & 0 & 0 & -1 \\
0 & 0 & \sqrt{-1} & 0 & 0 & -\sqrt{-1} & 0 \\
0 & 0 & 0 & -\sqrt{-1} & 0 & 0 & \sqrt{-1}
\end{array}\right) .
$$

Consider $f_{1}, \cdots, f_{7}$ in (2.9). Suppose $x=\sum_{i=1}^{7} x_{i} f_{i}=\phi(y)$. Then

$$
\left(\begin{array}{c}
y_{1} \\
\vdots \\
y_{7}
\end{array}\right)={ }^{t} g_{1}^{-1}\left(\begin{array}{c}
x_{1} \\
\vdots \\
x_{7}
\end{array}\right) \text {. }
$$

Note that $\operatorname{det} g_{1}=2^{3}$. So we put $t=2^{9}, g=2^{-3} g_{1}$ following the argument of Proposition (2.24). Then $t^{2} \operatorname{det} g g=g_{1}$.

Consider $(t, g) \in \mathrm{GL}_{\mathbb{C}}$. Since $t g v=2^{6} g_{1} v$ for $v \in W$, under $(t, g),\left(e_{1}, \cdots, e_{7}\right)$ maps to $2^{6}\left(e_{1}, \cdots, e_{7}\right) g_{1}$. By an easy computation,

$$
w_{1}=(t, g) w=2^{7}\left(e_{145}-e_{167}+e_{347}-e_{356}+e_{123}+e_{246}+e_{257}\right) .
$$

By the proof of Proposition (2.24), $w_{1}$ corresponds to $\mathbb{O}$. It is easy to see that

$$
\|\phi(y)\|_{w}=y_{1}^{2}+\cdots+y_{7}^{2} .
$$

Since $\Delta\left(w_{1}\right)=2^{9} \cdot 6, Q_{w_{1}}(y)=2^{9} \cdot 6\left(y_{1}^{2}+\cdots+y_{7}^{2}\right)$. Since $H_{w_{1}}^{\circ} \subset \operatorname{SO}\left(Q_{w_{1}}\right)$, the real rank of $H_{w_{1} \mathbb{R}}^{\circ}$ is zero.

\section{INTERMEDIATE GROUPS}

For $x \in V_{k}^{\text {ss }}$, let $\mathfrak{h}_{x}$ be the Lie algebra of $H_{x}^{\circ}$. Consider the element $w$ for cases (1)-(3). Let $\mathfrak{h}_{1}=\mathfrak{h}_{w}$ and $\mathfrak{h}_{2}=\operatorname{sl}(6)$, $\operatorname{sl}(7)$, or $\operatorname{sl}(2 n)$. In this section, we consider Lie subalgebras of $\mathfrak{h}_{2}$ containing $\mathfrak{h}_{1}$.

We first consider case (1). Clearly,

$$
\mathfrak{h}_{1}=\left\{\left(\begin{array}{cc}
A & 0 \\
0 & B
\end{array}\right) \mid \operatorname{tr}(A)=\operatorname{tr}(B)=0\right\} .
$$

Let

$$
\begin{aligned}
& \mathfrak{u}_{1}=\left\{\left(\begin{array}{cc}
0 & U \\
0 & 0
\end{array}\right) \mid U \in \mathrm{M}(3,3)\right\}, \\
& \mathfrak{u}_{2}=\left\{\left(\begin{array}{cc}
0 & 0 \\
U & 0
\end{array}\right) \mid U \in \mathrm{M}(3,3)\right\}, \\
& \mathfrak{t}=\left\{a\left(\begin{array}{cc}
I_{3} & 0 \\
0 & -I_{3}
\end{array}\right) \mid a \in k\right\} .
\end{aligned}
$$


Obviously $\mathfrak{h}_{1}$ is contained in the following Lie algebras

$$
\begin{aligned}
& \mathfrak{h}_{3}=\mathfrak{h}_{1} \oplus \mathfrak{u}_{1}, \mathfrak{h}_{4}=\mathfrak{h}_{1} \oplus \mathfrak{u}_{2}, \\
& \mathfrak{h}_{1}^{\prime}=\mathfrak{h}_{1} \oplus \mathfrak{t}, \mathfrak{h}_{3}^{\prime}=\mathfrak{h}_{1} \oplus \mathfrak{u}_{1} \oplus \mathfrak{t}, \mathfrak{h}_{4}^{\prime}=\mathfrak{h}_{1} \oplus \mathfrak{u}_{2} \oplus \mathfrak{t} .
\end{aligned}
$$

Proposition (3.4). If $\mathfrak{h}_{1} \subset \mathfrak{f} \subset \mathfrak{h}_{2}$ is a Lie subalgebra, $\mathfrak{f}=\mathfrak{h}_{1}, \mathfrak{h}_{1}^{\prime}, \mathfrak{h}_{2}, \mathfrak{h}_{3}, \mathfrak{h}_{3}^{\prime}, \mathfrak{h}_{4}$, or $\mathfrak{h}_{4}^{\prime}$.

Proof. As an $\mathfrak{h}_{1}$-module, $\mathfrak{h}_{2}$ decomposes as a direct sum of representations as

$$
\mathfrak{h}_{2}=\mathfrak{h}_{1} \oplus \mathfrak{u}_{1} \oplus \mathfrak{u}_{2} \oplus \mathfrak{t} .
$$

Let $\Lambda_{1}, \Lambda_{2}$ be the usual fundamental weights of sl(3), and $V_{1}, V_{2}$ the irreducible representations with highest weights $\Lambda_{1}, \Lambda_{2}$ respectively.

Then $\mathfrak{u}_{1} \cong V_{1} \otimes V_{2}, \mathfrak{u}_{2}=V_{2} \otimes V_{1}$, and $\mathfrak{t}$ is the trivial representation. Let $V_{3}$ be the representation of $\operatorname{sl}(3)$ with highest weight $\Lambda_{1}+\Lambda_{2}$. Let $V_{3,1}$ be the representation of $\mathfrak{h}_{1}$ which is the tensor product of $V_{3}$ for the first factor of sl(3) and the trivial representation for the second factor of $\operatorname{sl}(3)$. We define $V_{3,2}$ similarly. Then $\mathfrak{h}_{1}$ is $V_{3,1} \oplus V_{3,2}$ as a representation of $\mathfrak{h}_{1}$. No two of these irreducible representations are equivalent. So

$$
\mathfrak{f}=\mathfrak{h}_{1} \oplus\left(\mathfrak{f} \cap \mathfrak{u}_{1}\right) \oplus\left(\mathfrak{f} \cap \mathfrak{u}_{2}\right) \oplus(\mathfrak{f} \cap \mathfrak{t}) .
$$

Clearly, $\mathfrak{f} \cap \mathfrak{u}_{1}=\mathfrak{u}_{1}$ or 0 , etc. Since $\mathfrak{h}_{1}, \mathfrak{u}_{1}, \mathfrak{u}_{2}$ generate $\mathfrak{h}_{2}$, if $\mathfrak{f}$ contains both $\mathfrak{u}_{1}, \mathfrak{u}_{2}, \mathfrak{f}=\mathfrak{h}_{2}$. This proves the proposition.

We define $H_{w 1}=H_{w}^{\circ}, H_{w 2}=H$ and

$$
\begin{aligned}
& H_{w 3}=\left\{\left(\begin{array}{cc}
A & U \\
0 & B
\end{array}\right) \mid A, B \in \mathrm{SL}(3), U \in \mathrm{M}(3,3)\right\}, \\
& H_{w 4}=\left\{\left(\begin{array}{cc}
A & 0 \\
U & B
\end{array}\right) \mid A, B \in \mathrm{SL}(3), U \in \mathrm{M}(3,3)\right\} .
\end{aligned}
$$

If $k=\mathbb{R}$ and $x=g w$ for $g \in G_{\mathbb{R}}$, we define

$$
\begin{aligned}
& H_{x 1 \mathbb{R}}=H_{x \mathbb{R}+}^{\circ}, H_{x 2 \mathbb{R}}=H_{\mathbb{R}}, \\
& H_{x 3 \mathbb{R}}=g H_{w 3 \mathbb{R}} g^{-1}, H_{x 4 \mathbb{R}}=g H_{w 4 \mathbb{R}} g^{-1} .
\end{aligned}
$$

This definition does not depend on the choice of $g \in G_{\mathbb{R}}$.

Proposition (3.9). Suppose $x \in G_{\mathbb{R}} w$. Then if $H_{x 1 \mathbb{R}+} \subset F \subset H_{\mathbb{R}}$ is a closed connected subgroup whose radical is a unipotent subgroup, $F=H_{x 1 \mathbb{R}}, H_{x 2 \mathbb{R}}, H_{x 3 \mathbb{R}}$, or $H_{x 4 \mathbb{R}}$.

Proof. Let $\mathfrak{f}$ be the Lie algebra of $F$. Then $\mathfrak{h}_{1 \mathbb{R}} \subset g^{-1} \mathfrak{f} g \subset \mathfrak{h}_{2 \mathbb{R}}$. So $g^{-1} \mathfrak{f} g=$ $\mathfrak{h}_{1 \mathbb{R}}, \mathfrak{h}_{1 \mathbb{R}}^{\prime}, \mathfrak{h}_{2 \mathbb{R}}, \mathfrak{h}_{3 \mathbb{R}}, \mathfrak{h}_{4 \mathbb{R}}, \mathfrak{h}_{3 \mathbb{R}}^{\prime}$, or $\mathfrak{h}_{4 \mathbb{R}}^{\prime}$. But it cannot be $\mathfrak{h}_{1 \mathbb{R}}^{\prime}, \mathfrak{h}_{3 \mathbb{R}}^{\prime}$ or $\mathfrak{h}_{4 \mathbb{R}}^{\prime}$ because the radical of $F$ is a unipotent subgroup. This proves the proposition.

We consider the orbit of $w_{1}$ (see (1.13)) next. Let $g_{w_{1}}=g_{\sqrt{-1}}$ (see (1.8)).

Proposition (3.10). Let $x=g w_{1}$ for $g \in G_{\mathbb{R}}$. Then if $H_{x \mathbb{R}+}^{\circ} \subset F \subset H_{\mathbb{R}}$ is the identity component of the set of $\mathbb{R}$-rational points of an algebraic group defined over $\mathbb{Q}$ whose radical is a unipotent subgroup, $F=H_{x \mathbb{R}+}^{\circ}$ or $H_{\mathbb{R}}$. 
Proof. As in the previous proposition, we only have to consider the case $x=w_{1}$. Let $\mathfrak{f}$ be the Lie algebra of $F$, and $F_{\mathbb{C}} \subset H_{\mathbb{C}}$ the closed connected subgroup whose Lie algebra is $\mathfrak{f}_{\mathbb{C}}$. Then the radical of $F_{\mathbb{C}}$ is a unipotent subgroup and $F$ is the identity component of the set of $\mathbb{R}$-rational points of $F_{\mathbb{C}}$. So by a similar argument as in Proposition $(3.9), \mathfrak{f}_{\mathbb{C}}=\mathfrak{h}_{w_{1} 1 \mathbb{C}}, \mathfrak{h}_{w_{1} 2 \mathbb{C}}, \mathfrak{h}_{w_{1} 3 \mathbb{C}}$, or $\mathfrak{h}_{w_{1} 4 \mathbb{C}}$. We show that the last two cases cannot happen. Since the argument is similar, we only consider the case $\mathfrak{f}=\mathfrak{h}_{w_{1} 3 \mathbb{C}}$.

This implies that

$$
F_{\mathbb{C}}=\left\{g_{w_{1}}\left(\begin{array}{cc}
A & U \\
0 & B
\end{array}\right) g_{w_{1}}^{-1} \mid A, B \in \mathrm{SL}(3)_{\mathbb{C}}, U \in \mathrm{M}(3,3)_{\mathbb{C}}\right\} .
$$

The element $g_{w_{1}}\left(\begin{array}{cc}A & U \\ 0 & B\end{array}\right) g_{w_{1}}^{-1}$ is an $\mathbb{R}$-rational point if and only if $B=\bar{A}$ and $U=0$. So

$$
F=\left\{g_{w_{1}}\left(\begin{array}{cc}
A & 0 \\
0 & \bar{A}
\end{array}\right) g_{w_{1}}^{-1} \mid A \in \mathrm{SL}(3)_{\mathbb{C}}\right\}=H_{w_{1} \mathbb{R}+}^{\circ} .
$$

This implies $\mathfrak{f}=\mathfrak{h}_{w_{1} \mathbb{R}}$, which is a contradiction.

Next, we consider case (2). Let $\mathfrak{h}_{3}=\operatorname{so}\left(Q_{w}\right)$.

Proposition (3.11). Suppose $k$ is algebraically closed. Then if $\mathfrak{h}_{1} \subset \mathfrak{f} \subset \mathfrak{h}_{2}$ is a Lie subalgebra, $\mathfrak{f}=\mathfrak{h}_{1}, \mathfrak{h}_{2}$, or $\mathfrak{h}_{3}$.

Proof. Recall that $\mathfrak{h}_{1}$ is the simple Lie algebra of type $G_{2}$ and $\mathfrak{h}_{1} \subset \mathfrak{h}_{3} \subset \mathfrak{h}_{2}$. Let $W$ be the standard 7 dimensional representation of $\operatorname{sl}(7)$. As a representation of $\mathfrak{h}_{1}, W$ is irreducible and has highest weight equal to a fundamental weight $\Lambda_{1}$. There are no non-trivial representations of $\mathfrak{h}_{1}$ of lower dimension. The $\mathfrak{h}_{1}$-invariant complement to $\mathfrak{h}_{1}$ in $\mathfrak{h}_{3}$ is 7 dimensional and non-trivial (as $\mathfrak{h}_{3}$ is simple). Therefore $\mathfrak{h}_{3}=\mathfrak{h}_{1} \oplus W$. Now consider $\mathfrak{h}_{3} \subset \mathfrak{h}_{2}$. Let $\theta$ be the order two automorphism of $\mathfrak{h}_{2}$ defined by $\theta(X)=-{ }^{*} X$ (the adjoint with respect to $Q_{w}$ ). Then the fixed point set of $\theta$ is $\mathfrak{h}_{3}$. Write $\mathfrak{h}_{2}=\mathfrak{h}_{3} \oplus U$, the \pm 1 eigenspace decomposition for $\theta$. Then $[U, U]$ is contained in $\mathfrak{h}_{3}$ and is $\mathfrak{h}_{3}$-invariant, so $[U, U]=\mathfrak{h}_{3}$. Note that $\mathfrak{h}_{2} \cong W \otimes W^{*}$ and $W \cong W^{*}$, as $\mathfrak{h}_{2}-$ representations. Because the weight $2 \Lambda_{1}$ does not occur in $\mathfrak{h}_{3}$, this implies that the representation $U$ has highest weight $2 \Lambda_{1}$. The irreducible representation with highest weight $2 \Lambda_{1}$ has dimension 27 [14, p. 21], the same as $U$, so $U$ is irreducible. Thus, $\mathfrak{h}_{2}=\mathfrak{h}_{1} \oplus W \oplus U$, as representations of $\mathfrak{h}_{1}$.

Now suppose that $\mathfrak{f}$ is a subalgebra with $\mathfrak{h}_{1} \subset \mathfrak{f} \subset \mathfrak{h}_{2}$ with $\mathfrak{f} \neq \mathfrak{h}_{3}$. Then if $\mathfrak{f}$ contains $U$ it contains $\mathfrak{h}_{3}$, so must equal $\mathfrak{h}_{2}$. The proposition follows.

Corollary (3.12). Suppose $x \in V_{\mathbb{R}}^{\mathrm{ss}}$. Then if $H_{x \mathbb{R}+}^{\circ} \subset F \subset H_{\mathbb{R}}$ is a closed connected subgroup, $F=H_{x \mathbb{R}+}^{\circ}, \mathrm{SO}\left(Q_{x}\right)_{\mathbb{R}}$, or $H_{\mathbb{R}}$.

Proof. Let $\mathfrak{f}$ be the Lie algebra of $F$. Then $\mathfrak{h}_{x \mathbb{C}} \subset \mathfrak{f}_{\mathbb{C}} \subset \mathfrak{h}_{2 \mathbb{C}}$ and $\mathfrak{f}=\mathfrak{f}_{\mathbb{C}} \cap \mathfrak{h}_{2}$. Suppose $x=g w$ where $g \in G_{\mathbb{C}}$. Then

$$
g^{-1} \mathfrak{h}_{x \mathbb{C}} g=\mathfrak{h}_{1 \mathbb{C}} \subset g^{-1} \mathfrak{f}_{\mathbb{C}} g \subset \mathfrak{h}_{2 \mathbb{C}} .
$$

So $g^{-1} \mathfrak{f}_{\mathbb{C}} g=\mathfrak{h}_{1 \mathbb{C}}, \operatorname{so}\left(Q_{w}\right)_{\mathbb{C}}$, or $\mathfrak{h}_{2 \mathbb{C}}$. Therefore,

$$
\mathfrak{f}_{\mathbb{C}}=g \mathfrak{h}_{1 \mathbb{C}} g^{-1}=\mathfrak{h}_{x \mathbb{C}}, \quad g \operatorname{so}\left(Q_{w}\right)_{\mathbb{C}} g^{-1}=\operatorname{so}\left(Q_{x}\right)_{\mathbb{C}}, \text { or } \mathfrak{h}_{2 \mathbb{C}}
$$


This implies that

$$
\mathfrak{f}=\mathfrak{h}_{x \mathbb{R}}, \operatorname{so}\left(Q_{x}\right)_{\mathbb{R}}, \text { or } \mathfrak{h}_{2 \mathbb{R}}
$$

Finally, we consider case (3).

Proposition (3.13). If $H_{w \mathbb{R}+}^{\circ} \subset F \subset H_{\mathbb{R}}$ is a closed connected subgroup, $F=$ $H_{w \mathbb{R}+}^{\circ}$ or $H_{\mathbb{R}}$.

Proof. Let $\mathfrak{f}$ be the Lie algebra of $F$. Then $\mathfrak{h}_{1} \subset \mathfrak{f} \subset \mathfrak{h}_{2}$. Let $\Lambda_{1}, \Lambda_{2}$ be the first and the second fundamental weights of $\mathfrak{h}_{1}$. Then the standard representation $V_{1}=W$ of $\mathfrak{h}_{1}$ has the highest weight $\Lambda_{1}$. Let $V_{2}$ be the irreducible representation of $\mathfrak{h}_{1}$ with highest weight $\Lambda_{2}$. Since $w$ is a $G_{w}$-invariant non-degenerate bilinear form on $W^{*}, W$ is equivalent to $W^{*}$. So $\mathfrak{h}_{2}$ plus the trivial representation is equivalent to $W \otimes W$. Clearly $W \otimes W \cong \wedge^{2} W \oplus \operatorname{Sym}^{2} W$. It is known (see [14, pp. 14,15]) that $\wedge^{2} W$ is a sum of $V_{2}$ and the trivial representation. As a representation of $\mathfrak{h}_{1}, \mathfrak{h}_{1}$ is irreducible and has the same highest weight $2 \Lambda_{1}$ as $\operatorname{Sym}^{2} W$. By eliminating the trivial representation,

$$
\mathfrak{h}_{2}=\mathfrak{h}_{1} \oplus V_{2}
$$

Since $\mathfrak{h}_{1}, V_{2}$ are not equivalent, $\mathfrak{f}=\mathfrak{h}_{1} \oplus\left(\mathfrak{f} \cap V_{2}\right)$. So $\mathfrak{f}=\mathfrak{h}_{1}$ or $\mathfrak{h}_{2}$.

Since $V_{\mathbb{R}}^{\text {ss }}$ is a single $G_{\mathbb{R}}$-orbit in this case, the following is an immediate consequence of the above proposition.

Corollary (3.14). Suppose $x \in V_{\mathbb{R}}^{\mathrm{ss}}$. Then if $H_{x \mathbb{R}+}^{\circ} \subset F \subset H_{\mathbb{R}}$ is a closed connected subgroup, $F=H_{x \mathbb{R}+}^{\circ}$, or $H_{\mathbb{R}}$.

\section{The FIXED POINT SET OF $H_{x}^{\circ}$}

We consider the fixed point set of $H_{x}^{\circ}$ for $x \in V_{k}^{\text {ss }}$. Throughout this section, we assume that $k$ is algebraically closed.

We first consider case (1). Let $W$ be the standard representation of GL(6).

Proposition (4.1). If $y \in V_{k}$ is fixed by $H_{w k}^{\circ}$, there exist $\alpha_{1}, \alpha_{2} \in k$ such that $y=\alpha_{1} e_{123}+\alpha_{2} e_{456}$.

Proof. We use the notation of (3.1)-(3.6). Let $V_{1,1}$ be the representation of $\mathfrak{h}_{1}$ which is the tensor product of $V_{1}$ for the first factor and the trivial representation for the second factor. We define $V_{1,2}$ similarly. Then $W=V_{1,1} \oplus V_{1,2}$. Therefore,

$$
\wedge^{3} W \cong \wedge^{3} V_{1,1} \oplus\left(\wedge^{2} V_{1,1} \otimes V_{1,2}\right) \oplus\left(V_{1,1} \otimes \wedge^{2} V_{1,2}\right) \oplus \wedge^{3} V_{1,2} .
$$

The second and the third factors are irreducible and non-trivial, and the first and the fourth factors are the trivial representation. Therefore, the dimension of the subspace

$$
\left\{y \in V \mid g y=y \text { for all } g \in H_{w k}^{\circ}\right\}
$$

is two.

Obviously, $\alpha_{1} e_{123}+\alpha_{2} e_{456}$ belongs to the above space for all $\alpha_{1}, \alpha_{2} \in k$. This proves the proposition.

The following is an immediate consequence of the above proposition.

Corollary (4.3). Let $x \in V_{k}^{\mathrm{ss}}$. Then if $y \in V_{k}$ is fixed by $H_{x k}^{\circ}, \operatorname{Gr}(y)=\operatorname{Gr}(x)$. 
We consider case (2) next. Let $W$ be the standard representation of GL(7).

Proposition (4.4). If $y \in V_{k}$ is fixed by $H_{w k}^{\circ}, y$ is a scalar multiple of $w$.

Proof. We use the notation of Proposition (3.11). Recall that $H_{w}^{\circ}$ is a simple group of type $G_{2}$ and $W$ is the 7 dimensional irreducible representation with highest weight $\Lambda_{1}$. We show that the 35 dimensional representation $\wedge^{3} W$ contains the trivial representation exactly once. The weights of $W$ are the short roots of $\mathfrak{g}_{2}$, together with zero, so the non-zero weights form the vertices of a regular hexagon centered at 0 . Therefore, by listing the non-zero weights $\alpha_{1}, \cdots, \alpha_{6}$ in the order they appear around the hexagon (with $\alpha_{1}=\Lambda_{1}$ ), we have $\alpha_{i-1}+\alpha_{i+1}=\alpha_{i}$ for $1 \leq i \leq 6$ (with subscripts read modulo 6 ). So one sees that the highest possible weight of $\wedge^{3} W$ is $2 \Lambda_{1}$ (from the sum $\alpha_{6}+\alpha_{1}+\alpha_{2}$ ) and the multiplicity of the zero weight is 5 (from the three sums of the form $-\alpha_{i}+0+\alpha_{i}$ and the two sums of the form $\left.\alpha_{i}+\alpha_{i+2}+\alpha_{i+4}\right)$. Since the irreducible representation with highest weight $2 \Lambda_{1}$ has dimension 27 and must occur, 8 dimensions remain. As the smallest nontrivial representation of $G_{2}$ has dimension 7 , the only possibility is $W$ plus one copy of the trivial representation. Thus, $\wedge^{3} W=U \oplus W \oplus k$.

The following is an immediate consequence of the above proposition.

Corollary (4.5). Let $x \in V_{k}^{\mathrm{ss}}$. Then if $y \in V_{k}$ is fixed by $H_{x k}^{\circ}$, $y$ is a scalar multiple of $x$.

Finally we consider case (3). Let $W$ be the standard representation of $\operatorname{Sp}(2 n)$. As we pointed out in $\S 3, \wedge^{2} W$ is a sum of a non-trivial irreducible representation and the trivial representation. Therefore, the following proposition follows by an argument as above.

Proposition (4.6). Let $x \in V_{k}^{\mathrm{ss}}$. Then if $y \in V_{k}$ is fixed by $H_{x k}^{\circ}, y$ is a scalar multiple of $x$.

\section{The ORBIT ClOSURE}

In this section, we formulate irrationality conditions for $x \in V_{\mathbb{R}}^{\mathrm{ss}}$ for cases (1)(3) and prove that the orbit of $H_{x \mathbb{R}+}^{\circ}$ in $H_{\mathbb{R}} / \Gamma$ is dense if $x \in V_{\mathbb{R}}^{\mathrm{ss}}$ is sufficiently irrational.

Consider case (1). Let $G, V, W, w_{0}=w, w_{1}$, etc. be as in $\S 1$.

Definition (5.1)(1). A point $x \in G_{\mathbb{R}} w$ is sufficiently irrational if both $\left[E_{x 1}\right]$, $\left[E_{x 2}\right] \in \operatorname{Gr}(3,6)_{\mathbb{C}}$ are irrational and $\operatorname{Gr}(x) \in((\mathbb{Z} / 2 \mathbb{Z}) \backslash(\operatorname{Gr}(3,6) \times \operatorname{Gr}(3,6)))_{\mathbb{R}}$ is irrational. A point $x \in G_{\mathbb{R}} w_{1}$ is sufficiently irrational if

$$
\operatorname{Gr}(x) \in((\mathbb{Z} / 2 \mathbb{Z}) \backslash(\operatorname{Gr}(3,6) \times \operatorname{Gr}(3,6)))_{\mathbb{R}}
$$

is irrational.

Note that if $x \in G_{\mathbb{R}} w_{1}, E_{x 1}, E_{x 2}$ are complex conjugates of each other. So the irrationality of one of $\left[E_{x 1}\right],\left[E_{x 2}\right]$ implies the irrationality of the other. Also even though we cannot distinguish $\left[E_{x 1}\right],\left[E_{x 2}\right]$, if we say both are irrational, the statement makes sense.

Next consider case (2). Let $G, V, W$, etc. be as in $\S 2$.

Definition (5.1)(2). A point $x \in V_{\mathbb{R}}^{\text {ss }}$ is sufficiently irrational if $\left[Q_{x}\right] \in \mathbb{P}\left(\mathrm{Sym}^{2} W\right)_{\mathbb{R}}$ is irrational. 
Next consider case (3). Let $W$ be the standard representation of $\operatorname{GL}(2 n)$ and $V=\wedge^{2} W$.

Definition (5.1)(3). A point $x \in V_{\mathbb{R}}^{\text {ss }}$ is sufficiently irrational if $[x] \in \mathbb{P}(V)_{\mathbb{R}}$ is irrational.

Now we are ready to prove that the orbit of $H_{x \mathbb{R}+}^{\circ}$ is dense.

Theorem (5.2). Let $\Gamma \subset S L(W)_{\mathbb{R}}$ be an arithmetic lattice. Then if $x \in V_{\mathbb{R}}^{\mathrm{ss}}$ is sufficiently irrational in the sense of Definition (5.2)(1)-(3) and the real rank of $H_{x \mathbb{R}}^{\circ}$ is positive, $H_{x \mathbb{R}+}^{\circ} \Gamma$ is dense in $H_{\mathbb{R}}$.

Proof. Note that the real rank of $H_{x \mathbb{R}}^{\circ}$ is positive for all $x \in V_{\mathbb{R}}^{\text {ss }}$ for cases (1), (3), and for $x \in G_{\mathbb{R}} w$ for case (2).

The proof for case (2) is the same as the proof of Theorem (5.1) [20] (using Corollaries (3.12),(4.5)). The proof for case (3) is also similar using Corollary (3.14) and Proposition (4.6). So we only consider case (1).

By Theorem (0.2) (Ratner's theorem), there exists a connected closed subgroup $H_{x \mathbb{R}+}^{\circ} \subset F \subset \mathrm{SL}(W)_{\mathbb{R}}$ such that $\overline{H_{x \mathbb{R}+}^{\circ} \Gamma}=F \Gamma$. By Proposition (3.2) [16, pp. 321-322], $F$ is defined over $\mathbb{Q}$ and the radical of $F$ is a unipotent subgroup. So by Propositions (3.9), (3.10), $F=H_{x 1 \mathbb{R}}, H_{x 2 \mathbb{R}}, H_{x 3 \mathbb{R}}$, or $H_{x 4 \mathbb{R}}$.

Suppose $F=H_{x \mathbb{R}+}^{\circ}$. Since $F$ is defined over $\mathbb{Q}$, for any $\sigma \in \operatorname{Aut}(\mathbb{C} / \mathbb{Q}), F_{\mathbb{C}}^{\sigma}=F_{\mathbb{C}}$. So $H_{x^{\sigma} \mathbb{C}}^{\circ}=H_{x \mathbb{C}}^{\circ}=H_{x \mathbb{C}}^{\circ}$. Since this group fixes $x^{\sigma}$,

$$
\operatorname{Gr}(x)^{\sigma}=\operatorname{Gr}\left(x^{\sigma}\right)=\operatorname{Gr}(x)
$$

by Proposition (1.20) and Corollary (4.3). Since this is the case for all $\sigma, \operatorname{Gr}(x)$ is a $\mathbb{Q}$-rational point of $(\mathbb{Z} / 2 \mathbb{Z}) \backslash(\operatorname{Gr}(3,6) \times \operatorname{Gr}(3,6))$, which is a contradiction.

Suppose $F=H_{x 3 \mathbb{R}}$ or $H_{x 4 \mathbb{R}}$ (which means $x \in G_{\mathbb{R}} w$ ). Since the argument is similar, we only consider the case $F=H_{x 3 \mathbb{R}}$. Suppose $x=g w$ for $g \in \operatorname{GL}(W)_{\mathbb{C}}$. Then if $\sigma \in$ Aut $(\mathbb{C} / \mathbb{Q}), x^{\sigma}=g^{\sigma} w$ and

$$
\begin{aligned}
H_{x 3 \mathbb{C}}^{\sigma} & =\left\{g^{\sigma}\left(\begin{array}{cc}
A^{\sigma} & U^{\sigma} \\
0 & B^{\sigma}
\end{array}\right)\left(g^{\sigma}\right)^{-1} \mid A, B \in \mathrm{SL}(3)_{\mathbb{C}}, U \in \mathrm{M}(3,3)_{\mathbb{C}}\right\} \\
& =\left\{g^{\sigma}\left(\begin{array}{cc}
A & U \\
0 & B
\end{array}\right)\left(g^{\sigma}\right)^{-1} \mid A, B \in \mathrm{SL}(3)_{\mathbb{C}}, U \in \mathrm{M}(3,3)_{\mathbb{C}}\right\} \\
& =H_{x^{\sigma}} 3 \mathbb{C} .
\end{aligned}
$$

Since this group is defined over $\mathbb{Q}, H_{x 3 \mathbb{C}}=H_{x 3 \mathbb{C}}^{\sigma}=H_{x^{\sigma}} 3 \mathbb{C}$. Note that $\left[E_{w 1}\right] \in$ $\operatorname{Gr}(3,6)_{\mathbb{C}}$ is the unique point fixed by $H_{w 3 \mathbb{C}}$. Since $g\left[E_{w 1}\right]$ (resp. $\left.g^{\sigma}\left[E_{w 1}\right]\right)$ is fixed by $H_{x 3 \mathbb{C}}\left(\right.$ resp. $\left.H_{x^{\sigma}} 3 \mathbb{C}\right), g\left[E_{w 1}\right]=g^{\sigma}\left[E_{w 1}\right]=\left(g\left[E_{w 1}\right]\right)^{\sigma}$. Since this is the case for all $\sigma, g\left[E_{w 1}\right]$ must be a $\mathbb{Q}$-rational point of $\operatorname{Gr}(3,6)$. So either $\left[E_{x 1}\right]$ or $\left[E_{x 2}\right]$ is a $\mathbb{Q}-$ rational point of $\operatorname{Gr}(3,6)$, which is a contradiction. This proves that $F=H_{\mathbb{R}}$.

\section{Analogues of the Oppenheim conjecture}

We prove our main theorem of this paper in this section.

We consider case (1) in (6.1)-(6.6). Consider $V, W,\left\{e_{1}, \cdots, e_{6}\right\}, v, w_{0}=w, w_{1}$ in $\S 1$.

Proposition (6.1). Let $y=\left(y_{i j k}\right) \in \wedge^{3} \mathbb{R}^{5}$, and $\epsilon>0$. Then there exist $z_{0}=$ $\left(z_{0, i j k}\right) \in G_{\mathbb{R}} w_{0}$ and $z_{1}=\left(z_{1, i j k}\right) \in G_{\mathbb{R}} w_{1}$ such that $\left|y_{i j k}-z_{l, i j k}\right|<\epsilon$ for $l=$ $0,1,1 \leq i<j<k \leq 5$. 
Proof. Let $z=\left(z_{i j k}\right)$ and

$$
X=\left(\begin{array}{lll}
z_{234} & -z_{134} & z_{124} \\
z_{235} & -z_{135} & z_{125} \\
z_{236} & -z_{136} & z_{126}
\end{array}\right), Y=\left(\begin{array}{lll}
z_{156} & -z_{146} & z_{145} \\
z_{256} & -z_{246} & z_{245} \\
z_{356} & -z_{346} & z_{345}
\end{array}\right)
$$

Note that $z \in G_{\mathbb{R}} w_{0}$ (resp. $z \in G_{\mathbb{R}} w_{1}$ ) if and only if $\Delta(z)>0$ (resp. $\Delta(z)<0$ ). It is proved in [14, p. 83] that

$$
\begin{aligned}
\Delta(z)= & \left(z_{123} z_{456}-\operatorname{tr}(X Y)\right)^{2}+4 z_{123} \operatorname{det} Y \\
& +4 z_{123} \operatorname{det} X-4 \sum_{i, j} \operatorname{det} X_{i j} \operatorname{det} Y_{j i},
\end{aligned}
$$

where $X_{i j}, Y_{j i}$ are the $(i, j)$-minor and the $(j, i)$-minor of $X, Y$ respectively.

As a polynomial of $z_{456}$, this is a quadratic polynomial and the coefficient of $z_{456}^{2}$ is $z_{123}^{2}$. Given $y$, we can choose $z_{123} \neq 0$ close to $y_{123}$. By taking $z_{456} \gg 0$, $\Delta(z)>0$. So the existence of $z_{0}$ follows.

We try to choose $z_{1}$ so that $z_{1, i j 6}=0$ unless $(i, j)=(1,5),(2,4),(4,5)$. So we assume $z_{i j 6}=0$ unless $(i, j)=(1,5),(2,4),(4,5)$ in the following. We first choose $z_{123} \neq 0$ close to $y_{123}$. Then

$$
\begin{aligned}
\Delta(z)= & z_{123}^{2}\left(z_{456}-z_{123}^{-1} \operatorname{tr}(X Y)\right)^{2}+4 z_{123} \operatorname{det} Y \\
& +4\left(z_{456}-z_{123}^{-1} \operatorname{tr}(X Y)\right) \operatorname{det} X \\
& +4 z_{123}^{-1} \operatorname{tr}(X Y) \operatorname{det} X-4 \sum_{i, j} \operatorname{det} X_{i j} \operatorname{det} Y_{j i} .
\end{aligned}
$$

As a quadratic polynomial of $z_{456}-z_{123}^{-1} \operatorname{tr}(X Y),(6.4)$ can take a negative value if the discriminant is positive. Let

$$
f_{1}(z)=16 z_{123}^{2}\left(-z_{123} z_{345}+z_{234} z_{135}+z_{145} z_{235}\right) .
$$

Then a simple consideration shows that there exist polynomials $f_{2}(z), f_{3}(z), f_{4}(z)$ which depend only on $\left(z_{i j k}\right)_{i<j<k \leq 5}$ such that the the discriminant of (6.4) is

$$
f_{1}(z) z_{156} z_{246}+f_{2}(z) z_{156}+f_{3}(z) z_{246}+f_{4}(z) .
$$

Note that if $f$ is a non-zero polynomial on a real vector space $U_{\mathbb{R}}$, the set $\{p \in$ $\left.U_{\mathbb{R}} \mid f(p) \neq 0\right\}$ is open dense in $U_{\mathbb{R}}$ in the classical topology. Since $f_{1}(z)$ is not identically zero, we can choose $\left(z_{i j k}\right)_{i<j<k \leq 5}$ close to $y$ so that $f_{1}(z) \neq 0$. If $f_{1}(z)>0$, we choose $z_{156}=z_{246} \gg 0$. If $f_{1}(z)<0$, we choose $z_{156}=-z_{246} \gg 0$. In both cases, we can make (6.6) positive.

We consider case (2) in (6.7)-(6.13). Consider $V, W,\left\{e_{1}, \cdots, e_{7}\right\}, v, w$ in $\S 2$. We use the notation $e_{i_{1} \cdots i_{k}}=e_{i_{1}} \wedge \cdots \wedge e_{i_{k}}$ as before.

Proposition (6.7). Let $y=\left(y_{i j k}\right) \in \wedge^{3} \mathbb{R}^{6}$, and $\epsilon>0$. Then there exists $z=$ $\left(z_{i j k}\right) \in G_{\mathbb{R}} w$ such that $\left|y_{i j k}-z_{i j k}\right|<\epsilon$ for $1 \leq i<j<k \leq 6$. 
Proof. We try to choose $z$ so that $z \in V_{\mathbb{R}}^{\text {ss }}, Q_{z}\left(v_{1}\right)>0$, and $Q_{z}\left(v_{7}\right)<0$. This condition ensures that $Q_{z}$ is indefinite and therefore, $z \in G_{\mathbb{R}} w$. Let

$$
\begin{aligned}
& I_{1}=\left\{\left(j, k, j^{\prime}, k^{\prime}, j^{\prime \prime}, k^{\prime \prime}\right) \mid \begin{array}{c}
j<k, j^{\prime}<k^{\prime}, j^{\prime \prime}<k^{\prime \prime}, \\
\left\{j, k, j^{\prime}, k^{\prime}, j^{\prime \prime}, k^{\prime \prime}\right\}=\{2, \cdots, 7\}
\end{array}\right\}, \\
& I_{2}=\left\{\left(i, j, i^{\prime}, j^{\prime}, i^{\prime \prime}, j^{\prime \prime}\right) \mid \begin{array}{c}
i<j, i^{\prime}<j^{\prime}, i^{\prime \prime}<j^{\prime \prime}, \\
\left\{i, j, i^{\prime}, j^{\prime}, i^{\prime \prime}, j^{\prime \prime}\right\}=\{1, \cdots, 6\}
\end{array}\right\}, \\
& I_{3}=\left\{\left(j, k, j^{\prime}, k^{\prime}\right) \mid j<k, j^{\prime}<k^{\prime},\left\{j, k, j^{\prime}, k^{\prime}\right\}=\{3,4,5,6\}\right\} .
\end{aligned}
$$

Lemma (6.9). (1) The coefficient of $v_{1}^{2}$ in $Q_{z}$ is

$$
f_{1}(z)=\sum_{\left(j, k, j^{\prime}, k^{\prime}, j^{\prime \prime}, k^{\prime \prime}\right) \in I_{1}} \operatorname{sgn}\left(\begin{array}{ccc}
2 & \cdots & 7 \\
j & \cdots & k^{\prime \prime}
\end{array}\right) z_{1 j k} z_{1 j^{\prime} k^{\prime}} z_{1 j^{\prime \prime} k^{\prime \prime}}
$$

(2) The coefficient of $v_{7}^{2}$ in $Q_{z}$ is

$$
f_{2}(z)=\sum_{\left(i, j, i^{\prime}, j^{\prime}, i^{\prime \prime}, j^{\prime \prime}\right) \in I_{2}} \operatorname{sgn}\left(\begin{array}{ccc}
1 & \cdots & 6 \\
i & \cdots & j^{\prime \prime}
\end{array}\right) z_{i j 7} z_{i^{\prime} j^{\prime} 7} z_{i^{\prime \prime} j^{\prime \prime} 7}
$$

Proof. We consider (1) first. Consider $z \wedge D_{3}(z) \wedge D_{3}(z)$.

$$
D_{3}(z)=\sum_{i^{\prime}<j^{\prime}<k^{\prime}} z_{i^{\prime} j^{\prime} k^{\prime}}\left(e_{j^{\prime} k^{\prime}} \otimes e_{i^{\prime}}-e_{i^{\prime} k^{\prime}} \otimes e_{j^{\prime}}+e_{i^{\prime} j^{\prime}} \otimes e_{k^{\prime}}\right) .
$$

Since $j^{\prime}, k^{\prime}>1$, the terms in the right hand side of (6.10) which has $e_{1}$ as the second factor are those with $i^{\prime}=1$. Therefore,

$$
\begin{aligned}
z & \wedge D_{3}(z) \wedge D_{3}(z) \\
= & \left(\sum_{i, j, k} \sum_{j^{\prime}, k^{\prime}} \sum_{j^{\prime \prime}, k^{\prime \prime}} x_{i j k} x_{1 j^{\prime} k^{\prime}} x_{1 j^{\prime \prime} k^{\prime \prime}} e_{i j k} \wedge e_{j^{\prime} k^{\prime}} \wedge e_{j^{\prime \prime} k^{\prime \prime}}\right) \otimes e_{1} \otimes e_{1} \\
& +\cdots .
\end{aligned}
$$

Clearly, $e_{i j k} \wedge e_{j^{\prime} k^{\prime}} \wedge e_{j^{\prime \prime} k^{\prime \prime}}=0$ unless $i=1$ and $\left(j, k, j^{\prime}, k^{\prime}, j^{\prime \prime}, k^{\prime \prime}\right) \in I_{1}$. So we only have to consider indices in $I_{1}$. If $\left(j, k, j^{\prime}, k^{\prime}, j^{\prime \prime}, k^{\prime \prime}\right) \in I_{1}$,

$$
e_{1 j k} \wedge e_{j^{\prime} k^{\prime}} \wedge e_{j^{\prime \prime} k^{\prime \prime}}=\operatorname{sgn}\left(\begin{array}{ccc}
2 & \cdots & 7 \\
j & \cdots & k^{\prime \prime}
\end{array}\right) e_{1234567} .
$$

This proves (1).

Note that

$$
e_{i j 7} \wedge e_{i^{\prime} j^{\prime}} \wedge e_{i^{\prime \prime} j^{\prime \prime}}=e_{i j} \wedge e_{i^{\prime} j^{\prime}} \wedge e_{i^{\prime \prime} j^{\prime \prime}} \wedge e_{7}
$$

So (2) is similar.

We consider $z$ such that $z_{i j 7}=0$ unless $(i, j)=(1,2),(3,4),(5,6)$. Note that this has no effect on $z_{i j k}$ with $i<j<k \leq 6$. We define

$$
f_{3}(z)=\sum_{\left(j, k, j^{\prime}, k^{\prime}\right) \in I_{3}} \operatorname{sgn}\left(\begin{array}{ccc}
3 & \cdots & 6 \\
j & \cdots & k^{\prime}
\end{array}\right) z_{1 j k} z_{1 j^{\prime} k^{\prime}} .
$$

The following is an immediate consequence of the above lemma. 
Corollary (6.13). Suppose $z_{i j 7}=0$ unless $(i, j)=(1,2),(3,4),(5,6)$. Then there exists a polynomial $f_{4}(z)$ which does not depend on $z_{127}, z_{347}, z_{567}$ such that

$$
f_{1}(z)=3 z_{127} f_{3}(z)+f_{4}(z), f_{2}(z)=6 z_{127} z_{347} z_{567} .
$$

Since $f_{3}(z)$ is non-zero, we can choose $\left(z_{i j k}\right)_{i<j<k \leq 6}$ arbitrarily close to $y$ and $f_{3}(z) \neq 0$. If $f_{3}(z)>0$, we choose $z_{127} \gg 0, z_{347} z_{567}<0$. If $f_{3}(z)>0$, we choose $z_{127} \ll 0, z_{347} z_{567}>0$. In both cases, $f_{1}(z)>0, f_{2}(z)<0$. Since $V_{\mathbb{R}}^{\text {ss }}$ is open dense in $V_{\mathbb{R}}$, we can replace $z$ if necessary and assume that $z \in V_{\mathbb{R}}^{\text {ss }}$. In this process, the condition $f_{1}(z)>0, f_{2}(z)<0$ can be preserved. This completes the proof of Proposition (6.7).

Now we consider case (3). So $G=\operatorname{GL}(2 n), W=\mathbb{Q}^{2 n}, V=\wedge^{2} W$. In this case, $V_{\mathbb{R}}^{\text {ss }}$ is a single $G_{\mathbb{R}}$-orbit. So the following proposition is obvious.

Proposition (6.14). Let $y=\left(y_{i j}\right) \in \wedge^{2} \mathbb{R}^{2 n-1}$, and $\epsilon>0$. Then there exist $z=\left(z_{i j}\right) \in V_{\mathbb{R}}^{\mathrm{ss}}$ such that $\left|y_{i j}-z_{i j}\right|<\epsilon$ for $1 \leq i<j \leq 2 n-1$.

Now we are ready to prove our main theorem. In statements (1)-(3) of the following theorem, we consider the prehomogeneous vector spaces (1)-(3) of this paper respectively. Let $W$ be the standard representation of GL(6), GL(7), or GL $(2 n)$. We identify $\wedge^{3} W$ and $\wedge^{2} W$ with $\wedge^{3}\left(W^{*}\right)^{*}$ and $\wedge^{2}\left(W^{*}\right)^{*}$ respectively.

Theorem (6.15). Suppose $x \in V_{\mathbb{R}}^{\mathrm{ss}}$ is sufficiently irrational in the sense of Definition (5.1)(1)-(3) and the real rank of $H_{x \mathbb{R}}^{\circ}$ is positive.

(1) For any $y=\left(y_{i j k}\right) \in \wedge^{3} \mathbb{R}^{5}$ and $\epsilon>0$, there exists a $\mathbb{Z}$-basis $\left\{u_{1}, \cdots, u_{6}\right\}$ of $W_{\mathbb{Z}}^{*} \cong \mathbb{Z}^{6}$ such that

$$
\left|y_{i j k}-x\left(u_{i}, u_{j}, u_{k}\right)\right|<\epsilon
$$

for all $i<j<k \leq 5$.

(2) For any $y=\left(y_{i j k}\right) \in \wedge^{3} \mathbb{R}^{6}$ and $\epsilon>0$, there exists a $\mathbb{Z}$-basis $\left\{u_{1}, \cdots, u_{7}\right\}$ of $W_{\mathbb{Z}}^{*} \cong \mathbb{Z}^{7}$ such that

$$
\left|y_{i j k}-x\left(u_{i}, u_{j}, u_{k}\right)\right|<\epsilon
$$

for all $i<j<k \leq 6$.

(3) For any $y=\left(y_{i j}\right) \in \wedge^{2} \mathbb{R}^{2 n-1}$ and $\epsilon>0$, there exists a $\mathbb{Z}$-basis $\left\{u_{1}, \cdots, u_{2 n}\right\}$ of $W_{\mathbb{Z}}^{*} \cong \mathbb{Z}^{2 n}$ such that

$$
\left|y_{i j}-x\left(u_{i}, u_{j}\right)\right|<\epsilon
$$

for all $i<j \leq 2 n-1$.

Proof. Since the proof is similar we only consider case (1). For cases (2), (3) the argument is similar using Theorem (5.2) and Propositions (6.7), (6.14).

By Proposition (6.1), we can choose $z=\left(z_{i j k}\right) \in G_{\mathbb{R}} x$ such that $\left|y_{i j k}-z_{l, i j k}\right|<\frac{\epsilon}{2}$ for $l=0,1,1 \leq i<j<k \leq 5$. Since any element of $G_{\mathbb{R}}$ can be written as a product of an element of $H_{\mathbb{R}}$ and a scalar matrix, we can choose $h^{\prime} \in H_{\mathbb{R}}$ and $\lambda \in \mathbb{R} \backslash\{0\}$ so that $h^{\prime-1} x=\lambda z$. Let

$$
h=h^{\prime}\left(\begin{array}{cccc}
\lambda^{\frac{1}{3}} & & & \\
& \ddots & & \\
& & \lambda^{\frac{1}{3}} & \\
& & & \lambda^{-\frac{5}{3}}
\end{array}\right) \in H_{\mathbb{R}} .
$$

We put $z^{\prime}=h^{-1} x$. Then if $z^{\prime}=\left(z_{i j k}^{\prime}\right), z_{i j k}^{\prime}=z_{i j k}$ for all $i<j<k \leq 5$. 
By Theorem $(5.2), H_{x \mathbb{R}+}^{\circ} H_{\mathbb{Z}}$ is dense in $H_{\mathbb{R}}$. So we can choose $h_{1} \in H_{x \mathbb{R}+}^{\circ}$ and $h_{2} \in H_{\mathbb{Z}}$ so that $h_{1} h_{2}$ is close to $h$. Then $\left(h_{1} h_{2}\right)^{-1} x=h_{2}^{-1} h_{1}^{-1} x=h_{2}^{-1} x$ is close to $z^{\prime}=h^{-1} x$. Let $f_{1}, \cdots, f_{6}$ be the standard coordinate vectors of $W^{*}$. Since $h_{2}^{-1} x$ is close to $z^{\prime}$, we can assume that

$$
\left|z^{\prime}\left(f_{i}, f_{j}, f_{k}\right)-h_{2}^{-1} x\left(f_{i}, f_{j}, f_{k}\right)\right|=\left|z_{i j k}-x\left(h_{2} f_{i}, h_{2} f_{j}, h_{2} f_{k}\right)\right|<\frac{\epsilon}{2}
$$

for all $i<j<k \leq 5$.

Let $u_{1}=h_{2} f_{1}, \cdots, u_{6}=h_{2} f_{6}$. Since $h_{2} \in H_{\mathbb{Z}},\left\{u_{1}, \cdots, u_{6}\right\}$ is a $\mathbb{Z}$-basis of $W_{\mathbb{Z}}^{*}$. Moreover,

$$
\left|y_{i j k}-x\left(u_{i}, u_{j}, u_{k}\right)\right| \leq\left|y_{i j k}-z_{i j k}\right|+\left|z_{i j k}-x\left(u_{i}, u_{j}, u_{k}\right)\right|<\epsilon
$$

for all $i<j<k \leq 5$. This proves the theorem.

\section{NOTE ADDED IN PROOF}

The authors belatedly realized that the proof of Proposition (2.24) is slightly incorrect and the following adjustment has to be made.

(1) In the first part of the proof of Proposition (2.24), using the assumption that $g^{\prime}$ is compatible with the product and the norm, one can deduce that

$$
g^{\prime-1} y\left(v_{1}, v_{2}, v_{3}\right)=\Delta(x)^{-1} \Delta(y) x\left(v_{1}, v_{2}, v_{3}\right) .
$$

This implies that $y=\left(\Delta(x)^{-1} \Delta(y), g^{\prime}\right) x \in G_{k} x$.

(2) In the second part of the proof of Proposition (2.24), we have to put $x=$ $\left(1, g^{\prime}\right) w$. It is easy to see that $x \in V_{k}^{\mathrm{ss}}$ and $m_{w, x,\left(1, g^{\prime}\right)}(v)=\operatorname{det} g^{\prime} g^{\prime} v$. Then the $k$-form of $\operatorname{Im}(\widetilde{\mathbb{O}})$ which corresponds to $x$ is

$$
\begin{aligned}
\{v \in & \left.\operatorname{Im}(\widetilde{\mathbb{O}})_{\bar{k}} \mid \operatorname{det} g^{\prime} g^{\prime} v \in \operatorname{Im}(\widetilde{\mathbb{O}})_{k}\right\} \\
& =\left\{v \in \operatorname{Im}(\widetilde{\mathbb{O}})_{\bar{k}} \mid g^{\prime} v \in \operatorname{Im}(\widetilde{\mathbb{O}})_{k}\right\} \\
& =\operatorname{Im}(A)_{k} .
\end{aligned}
$$

Note that $\operatorname{det} g^{\prime} \in k^{\times}$.

The point is that given $g^{\prime} \in \mathrm{GL}(W)$, if we put $t=\left(\operatorname{det} g^{\prime}\right)^{3}, g=\left(\operatorname{det} g^{\prime}\right)^{-1} g^{\prime}$ then $t^{2} \operatorname{det} g g=\left(\operatorname{det} g^{\prime}\right)^{-1} g^{\prime}$ (note that multiplying a scalar to $g^{\prime}$ has the effect of multiplying its inverse when applied to $W^{*}$ ). In the text, we stated that this is $g^{\prime}$, and that's why we needed the above adjustment.

(3) At the end of $\S 2$, if we follow the above argument, we put $w_{1}=\left(1, g_{1}\right) w$. Then (2.26) must be replaced by

$$
w_{1}=2\left(w_{145}-e_{167}+e_{347}-e_{356}+3_{123}+e_{246}+e_{257}\right) .
$$

Also

$$
Q_{w_{1}}(y)=2^{3} Q_{w}\left({ }^{t} g_{1} y\right)=2^{3} \cdot 6\left(y_{1}^{2}+\cdots+y_{7}^{2}\right)
$$

and so $H_{w_{1}}^{\mathrm{o}} \subset \mathrm{SO}\left(Q w_{1}\right)$ is still of real rank zero.

\section{REFERENCES}

1. Maple V. Waterloo Maple Inc., Waterloo, Ontario, 1994.

2. Dani, S.G. Invariant measures and minimal sets of holospherical flows. Invent. Math., 64:357385, 1981. MR 83c:22009

3. Harvey, F.R. Spinors and calibrations. Academic Press, New York, San Francisco, London, 1990. MR 91e:53056 
4. Igusa, J. On a certain class of prehomogeneous vector spaces. J. of Pure and Applied Algebra, 47:265-282, 1987. MR 88m:20089

5. Kable, A.C., and A. Yukie. Prehomogeneous vector spaces and field extensions II. Invent. Math. 130 (1997), 315-344. CMP 98:02

6. Margulis, G.A. Lie groups and ergodic theory. In Avramov, L.L., and K. B. Tchakerian, editor, Algebra - Some current trends, Proceedings Varna 1986, volume 1352 of Lecture Notes in Mathematics, pages 130-146, Berlin, Heidelberg, New York, 1988. Springer-Verlag. MR 91a:22009

7. Margulis, G.A. Discrete subgroups and ergodic theory. In Number theory, trace formula and discrete groups, Symposium in honor of A. Selberg, Oslo 1987, pages 377-398, New York, San Francisco, London, 1989. Academic Press. MR 90k:22013a

8. Mumford, D. Lectures on curves on an algebraic surface, volume 59 of Annales of Mathematical Studies. Princeton University Press, Princeton, New Jersey, 1966. MR 35:187

9. Ratner, M. On measure rigidity of unipotent subgroups of semi-simple groups. Acta Math., 165:229-309, 1990. MR 91m:57031

10. Ratner, M. Strict measure rigidity for unipotent subgroups of solvable groups. Invent. Math., 101:449-482, 1990. MR 92h:22015

11. Ratner, M. On Raghunathan's measure conjecture. Ann. of Math., 134:545-607, 1991. MR 93a:22009

12. Ratner, M. On Raghunathan's topological conjecture and distributions of unipotent flows. Duke Math. J., 63:253-280, 1991. MR 93f:22012

13. Ratner, M. Invariant measures and orbit closures for unipotent actions on homogeneous spaces. Geom. Funct. Anal., 4:236-257, 1994. MR 95c:22018

14. Sato, M., and T. Kimura. A classification of irreducible prehomogeneous vector spaces and their relative invariants. Nagoya Math. J., 65:1-155, 1977. [MR 55:3341]

15. Sato, M., and T. Shintani. On zeta functions associated with prehomogeneous vector spaces. Ann. of Math., 100:131-170, 1974. MR 49:8969]

16. Shah, N. Uniformly distributed orbits of certain flows on homogeneous spaces. Math. Ann., 289:315-334, 1991. MR 93d:22010

17. Shintani, T. On Dirichlet series whose coefficients are class-numbers of integral binary cubic forms. J. Math. Soc. Japan, 24:132-188, 1972. MR 44:6619

18. Wright, D.J., and A. Yukie. Prehomogeneous vector spaces and field extensions. Invent. Math., 110:283-314, 1992. MR 93j:12004

19. Yukie, A. Shintani zeta functions, volume 183 of London Math. Soc. Lecture Note Series. Cambridge University Press, Cambridge, 1993. MR 95k:11037

20. Yukie, A. Prehomogeneous vector spaces and ergodic theory I. Duke Math. J. 90 (1997), 123-147. CMP 98:03

Department of Mathematics, Oklahoma State University, 401 Math Science, StillWATER, OKLAHOMa 74078-1058

E-mail address: dwitte@math.okstate.edu

Department of Mathematics, Oklahoma State University, 401 Math Science, StillWATER, OKLAHOMA 74078-1058

Current address: Mathematical Institute, Tôhoku University, Sendai Miyagi 980-8578, Japan

E-mail address: yukie@math.tohoku.ac.jp

$U R L:$ http:www.math.tohoku.ac.jp/ yukie

Department of Mathematics, Oklahoma State University, 401 Math Science, StillWATER, OKLAHOMA 74078-1058

E-mail address: zierau@math.okstate.edu 